\title{
Optical Spectroscopy of Embedded Young Stars in the Taurus-Auriga Molecular Cloud
}

\author{
Scott J. Kenyon \\ Harvard-Smithsonian Center for Astrophysics, 60 Garden Street, Cambridge, MA 02138 \\ e-mail: skenyon@cfa.harvard.edu \\ David I. Brown \\ Jet Propulsion Laboratory, MS 156-205, 4800 Oak Grove Drive, Pasadena, CA 91109 \\ e-mail: dib@lb6.jpl.nasa.gov \\ Christopher A. Tout \\ Institute of Astronomy, Madingley Road, Cambridge CB3 0HA England \\ e-mail: cat@ast.cam.ac.uk \\ and \\ Perry Berlind \\ Whipple Observatory, PO Box 97, Amado, AZ 85645 \\ e-mail: pberlind@cfa.harvard.edu \\ to appear in \\ The Astronomical Journal \\ June 1998
}




\begin{abstract}
This paper describes the first optical spectroscopic survey of class I sources (also known as embedded sources and protostars) in the Taurus-Auriga dark cloud. We detect 10 of the 24 known class I sources in the cloud at 5500-9000 $\AA$. All detected class I sources have strong $\mathrm{H} \alpha$ emission; most also have strong [O I] and [S II] emission. These data - together with high quality optical spectra of $\mathrm{T}$ Tauri stars in the Taurus-Auriga cloud - demonstrate that forbidden emission lines are stronger and more common in class I sources than in T Tauri stars. Our results also provide a clear discriminant in the frequency of forbidden line emission between weak-emission and classical $\mathrm{T}$ Tauri stars. In addition to strong emission lines, three class I sources have prominent $\mathrm{TiO}$ absorption bands. The M-type central stars of these sources mingle with optically visible $\mathrm{T}$ Tauri stars in the HR diagram and lie somewhat below both the birthline for spherical accretion and the deuterium burning sequence for disc accretion.
\end{abstract}

Subject headings: stars: pre-main-sequence - stars: formation - ISM: jets and outflows

\title{
1. INTRODUCTION
}

Examining the earliest phases of low mass stellar evolution requires observations of protostars deeply embedded in the dense cores of nearby molecular clouds. These "class I" sources (Lada 1987) have blackbody-like spectral energy distributions that peak at wavelengths of 30-100 $\mu \mathrm{m}$ and bolometric luminosities, $L_{\mathrm{b}} \approx 0.1-100 \mathrm{~L}_{\odot}$ (Adams et al. 1987; Myers et al. 1987; Wilking et al. 1989; Kenyon et al. 1990; Greene et al. 1994). Despite many detailed studies of their circumstellar environments (see, for example, Tamura et al. 1991a, b; André et al. 1993; Terebey et al. 1993; Moriarty-Schieven et al. 1992, 1995; Bontemps et al. 1996; Gómez et al. 1997; Hogerheijde et al. 1997; Whitney et al. 1997), understanding the stellar physics of these objects has proved elusive. Comparisons of observed bolometric luminosity functions with models is straightforward but controversial (Wilking et al. 1989; Kenyon et al. 1990; Fletcher \& Stahler 1994a, 1994b). The apparent lack of photospheric features in many objects has led several groups to abandon the HR diagram as a means for testing stellar evolutionary tracks of the youngest stars. These groups have proposed the bolometric temperature (Myers et al. 1998 and references therein), the submillimeter flux (Saraceno et al. 1996), and the visual extinction (Adams 1990) to replace effective temperature and have developed models to place evolving pre-main-sequence stars in their modified evolutionary diagrams. The accuracy of these techniques remains uncertain, because the methods are new and incompletely tested.

In this paper, we report an optical spectroscopic survey designed to detect photospheric absorption features from the central stars of class I sources in the Taurus-Auriga cloud. 
Although the line-of-sight extinction to the central star is large, $A_{V} \approx 30-60$ mag (Whitney et al. 1997), large ground-based telescopes can detect optical light scattered off cavities in the infalling envelopes of many objects. Optical data also provide the best measure of spectral types for pre-main-sequence stars. In general, I-band and J-band data are least contaminated by emission from an accretion disc and its associated boundary layer or magnetic accretion column (Kenyon \& Hartmann 1990). However, the very large continuum veiling detected on near-IR spectra of some class I sources (Casali \& Matthews 1992; Greene \& Lada 1996a, 1996b) favors I-band spectra, because the J-band veiling can be large if the disc extends to the stellar photosphere (Kenyon \& Hartmann 1990; Kenyon et al. 1996). Finally, optical spectra of class I sources allow an unambiguous comparison with optically brighter T Tauri stars, which have known spectral types in a well-calibrated system (see, for example, Kenyon \& Hartmann 1995; KH95 hereafter).

Our results provide the first optical detection of M-type absorption features in an embedded protostar. We identify $\mathrm{TiO}$ absorption bands in three Taurus-Auriga class I sources; one other star may have TiO features and a fifth star may have K-type absorption features. We use optical spectra of T Tauri stars to calibrate the spectral types of class I sources and then construct a complete HR diagram for the Taurus-Auriga cloud. These data, coupled with new evolutionary tracks for protostars accreting from discs and two spectral types derived from near-IR spectra (Greene \& Lada 1996b), show that class I sources in Taurus-Auriga mingle with T Tauri stars and lie below the birthline in the HR diagram.

We also detect strong emission lines on the spectra of all protostars. Forbidden emission from $[\mathrm{N} \mathrm{II}]$ and $[\mathrm{S} \mathrm{II}]$ is much more common among class I sources than older, optically brighter stars having the same bolometric luminosity. The fluxes of forbidden emission lines also seem stronger among class I sources than other pre-main-sequence stars in the cloud. We find no evidence that the permitted emission lines, such as $\mathrm{H} \alpha$ and He I, are more common or stronger than in T Tauri stars. These results extend and confirm previous conclusions that jet activity declines as a pre-main-sequence star contracts to the main-sequence.

We describe our observations in Sec. 2, explain our results in Sec. 3, and conclude with a brief discussion in Sec. 4.

\section{OBSERVATIONS}

We acquired optical spectra of faint Taurus-Auriga class I sources and other pre-mainsequence stars with the Red Channel Spectrograph at the Multiple Mirror Telescope (MMT; 16-19 November 1995) and the Double Spectrograph at the Palomar 5-m telescope (29-30 November 1995). At the MMT, we used the $270 \mathrm{~g} \mathrm{~mm}^{-1}$ grating and a $1^{\prime \prime}$ slit to produce spectra covering 5700-9000 $\AA$ on a $1200 \times 800$ Loral CCD. On-chip binning of the pixels, $2 \times 2$, yielded a spectral resolution of $10.8 \AA$ and a spatial resolution of 0 "' 6 per pixel. At 
Palomar, we used a $316 \mathrm{~g} \mathrm{~mm}^{-1}$ grating, a $2^{\prime \prime}$ slit, and a $1024 \times 1024 \mathrm{CCD}$. The Palomar spectra cover $6000-8500 \AA$ with a spectral resolution of $10 \AA$ and a spatial resolution of 0." 47 per pixel.

We obtained low resolution spectra of brighter young stars in Taurus-Auriga during 1995-1996 with FAST, a high throughput, slit spectrograph mounted at the Fred L. Whipple Observatory 1.5-m telescope on Mount Hopkins, Arizona (Fabricant et al. 1998). We used a $300 \mathrm{~g} \mathrm{~mm}^{-1}$ grating blazed at $4750 \AA$, a $3^{\prime \prime}$ slit, and recorded the spectra on a thinned Loral $512 \times 2688$ CCD. These spectra cover 3800-7500 $\AA$ at a resolution of $\sim 6 \AA$.

We derive final object spectra using standard tasks within NOAO IRAF. After trimming the CCD frames at each end of the slit, we correct for the bias level, flat-field each frame, apply an illumination correction, and derive a full wavelength solution from calibration lamps acquired immediately after each exposure. The wavelength solution for each frame has a probable error of $\pm 0.5-1.0 \AA$. We extract object and sky spectra using the optimal extraction algorithm within APEXTRACT. We vary the size of the object/sky aperture from source to source to include additional radiation from extended emission.

At both the MMT and Palomar, we selected class I sources based on published VRI photometry (see KH95), POSS red plates, and red narrow band continuum images Gómez et al. 1997). Our sample of 10 class I sources is complete to $\mathrm{R} \sim 20$ : we detected all sources with optical counterparts on the POSS and several sources with measured $R \sim 19-20$. We did not observe 14 other class I sources without known optical counterparts and cannot estimate a reliable detection frequency for deeply embedded sources. The observed sample spans the observed range of class I luminosities in the Taurus-Auriga cloud, $L_{\mathrm{b}} \sim 0.2-20$ $\mathrm{L}_{\odot}$, but does not include the reddest systems that do not have optical counterparts (see Whitney et al. 1997).

We selected FAST sources from the KH95 sample of $\sim 150$ known pre-main-sequence stars in the Taurus-Auriga cloud. We observed essentially all targets with $\mathrm{V} \lesssim 16-17$ : 55 out of 65 weak emission T Tauri stars, 69 out of 96 classical T Tauri stars, and one class I source with a bright optical counterpart (Haro 6-28). Aside from their apparent brightness, these samples do not appear to be biased against any particular observational property of pre-main-sequence stars: the distributions of $\mathrm{H} \alpha$ equivalent widths, $\mathrm{K}-\mathrm{L}$ colors, and bolometric luminosities of stars in the FAST sample are indistinguishable from the distributions for stars not included in the FAST sample using data from Cohen \& Kuhi (1979) and KH95. We thus conclude that the FAST objects are a representative sample of known pre-main-sequence stars in the cloud.

\section{RESULTS}




\subsection{Basic Properties and Spectral Types}

Figure 1 shows FAST spectra of T Tauri stars with a range of emission characteristics. Weak emission T Tauri stars, such as LkCa 3, have absorption spectra similar to normal main-sequence stars with additional weak $\mathrm{H} \alpha$ emission lines (Cohen \& Kuhi 1979; Walter et al. 1987). Classical T Tauri stars, such as BP Tau, DG Tau, and DP Tau, have prominent emission lines and a variable blue continuum superimposed on a late-type absorption spectrum (Cohen \& Kuhi 1979; Hartigan et al. 1989, 1991). Some T Tauri stars have few emission lines other than H I and He I; others have prominent [O I], [S II], and [Fe II] emission lines (Cohen \& Kuhi 1979). In most interpretations, the H I and He I lines form in an accretion region or outflowing wind close to the central star; the [O I] and [S II] lines form in a jet or in the wind (Basri \& Bertout 1993; Edwards et al. 1987; Hamann \& Persson 1992; see also Martin 1997).

Figure 2 shows contour maps of MMT spectra for several sources centered on $\mathrm{H} \alpha$ and [S II] $\lambda \lambda 6717,6730$. We fit gaussian profiles to the continuum and a few emission lines along the spatial direction for each class I source and three point sources. Several class I sources - such as L1489 IRS (04016+2610), HH31 IRS2 $(04248+2612)$, and 04264+2433 are clearly extended along the slit, with $\sigma \approx 5^{\prime \prime}-10^{\prime \prime}$ compared to $\sigma \approx 1^{\prime \prime} 0 \pm 0$ ". 2 for point sources. These sizes are comparable to sizes inferred from optical and near-infrared images of these sources (Whitney et al. 1997; Gómez et al. 1997, and references therein). The very deeply embedded sources L1527 IRS $(04368+2557)$ and L1551 IRS5 $(04287+1801)$ are much more extended than other class I sources. Both have large optical reflection nebulae, Z 30" across, with multiple emission knots (see, for example, Stocke et al. 1988; Graham \& Rubin 1992; Eiroa et al. 1994; Gómez et al. 1997).

Our results indicate that the continuum and emission lines of all class I sources are equally extended within the errors of the fit. The mean difference in spatial extent between the emission lines and the continuum is $\langle\sigma\rangle=0 . \prime 4 \pm 0.3$ for the 5 sources with strong continua (L1489 IRS, 04158+2805, HH31 IRS2, 04264+2433, and 04489+3042). This difference is small compared to the typical spatial extent of each source, $\sigma \approx 5^{\prime \prime}-10^{\prime \prime}$. Our observations have insufficient spatial resolution to discriminate between sources with emission knots such as L1527 IRS and those without emission knots such as 04489+3042.

Figures 3-5 show MMT and Palomar spectra for Taurus class I sources. We detect a strong continuum in several objects (Figure 3), including L1489 IRS, HH31 IRS2, $04264+2433$, and $04489+3042$. Three class I sources $-04158+2805,04248+2612$ and $04489+3042$ - have the deep TiO absorption bands characteristic of M-type stars. The continua of L1489 IRS and 04264+2433 appear featureless, although both have a prominent dip at $8100 \AA$. All of these class I sources have a strong $\mathrm{H} \alpha$ emission line, along with moderately strong emission from [O I], [S II], and Ca II. Several have He I emission at $\lambda \lambda 5876,6678,7065$. These spectra are similar to the spectra of classical T Tauri stars in Figure 1.

The spectra of class I sources in Figure 5 more closely resemble spectra of jets or 
Herbig-Haro (HH) objects (e.g., Böhm \& Solf; Dopita et al. 1982; Goodrich 1986; Reipurth \& Heathcote 1991; see also Raga et al. 1996). We detect little, if any, continuum emission from $04239+2436,04295+2251$, and L1527 IRS, but all of these objects have very strong emission lines (Figure 5). The relative intensities of the emission lines in $04239+2436$ and L1527 IRS are similar to those for on-source emission in HH30 IRS (Figure 4; right panel) and L1551 IRS5 (Figure 5; upper right panel). We detect [O I] and [S II] emission in all class I sources except 04295+2251 (Figure 5; lower left panel), where we identify a prominent $\mathrm{H} \alpha$ emission line superimposed on a very weak continuum.

To compare spectra of class I sources and T Tauri stars in more detail, we measure the strengths of several prominent absorption and emission lines. We fit gaussian profiles to obvious emission lines using SPLOT within NOAO IRAF and use the deblend option for blended lines such as the $[\mathrm{S} \mathrm{II}]$ doublet and the He I $\lambda 5876$ and $\mathrm{Na}$ I blend. In the absence of accurate de-reddened fluxes, the equivalent width, EW, provides a good relative measure of emission line strengths for strong continuum sources. We place upper limits of 100-200 $\AA$ for equivalent widths of weak continuum sources depending on the continuum level. Table 1 lists our results for class I sources; Table 2 summarizes measurements for FAST spectra of T Tauri stars. We include separate entries for the MMT and Palomar spectra of two objects, L1489 IRS and HH31 IRS2, and list the MMT results first in both cases. We estimate probable errors of $\pm 10 \%$ for strong lines with EW $>10 \AA$ and $\pm 20 \%$ for weaker lines based on measurements of 2-3 separate spectra for each star in the FAST sample and two stars in the MMT/Palomar sample.

We measure spectral types using $\mathrm{TiO}$ absorption indices, defined as the depth of a $\mathrm{TiO}$ band at a wavelength $\lambda$ relative to an interpolated continuum point (O'Connell 1973). The TiO bands at $\lambda 6180$ and $\lambda 7100$ are temperature-sensitive for M dwarfs and giants (O’Connell 1973). Kenyon \& Fernández-Castro (1987) derive reliable spectral types for the red giant components in symbiotic stars - which also have strong emission lines and a variable blue continuum - with these features. We define:

$$
[\mathrm{TiO}]_{1}=-2.5 \log \left(\frac{\mathrm{F}_{6180}}{\mathrm{~F}_{6125}+0.225\left(\mathrm{~F}_{6370}-\mathrm{F}_{6125}\right)}\right)
$$

and

$$
[\mathrm{TiO}]_{2}=-2.5 \log \left(\frac{\mathrm{F}_{7100}}{\mathrm{~F}_{7025}+0.2\left(\mathrm{~F}_{7400}-\mathrm{F}_{7025}\right)}\right)
$$

Each $30 \AA$ bandpass used for these indices avoids contamination from strong emission lines and telluric absorption bands. The two TiO indices increase from $[\mathrm{TiO}]_{1} \approx[\mathrm{TiO}]_{2} \approx 0$ at $\mathrm{K} 4-\mathrm{K} 5$ spectral types to $[\mathrm{TiO}]_{1} \approx 0.8$ and $[\mathrm{TiO}]_{2} \approx 1.0$ at M6 spectral types.

Figure 6 shows $[\mathrm{TiO}]_{2}$ as a function of $[\mathrm{TiO}]_{1}$ for normal main-sequence stars (filled circles), T Tauri stars with weak emission lines on FAST spectra (light triangles), and 
pre-main sequence stars with MMT or Palomar spectra (crosses and diamonds). The locus of $\mathrm{T}$ Tauri stars generally follows the main-sequence stars except near $[\mathrm{TiO}]_{1} \approx 0.5$, where pre-main-sequence stars have larger $[\mathrm{TiO}]_{2}$ indices compared to main-sequence stars. Most pre-main-sequence stars with MMT or Palomar spectra lie on the T Tauri star locus. Both L1489 IRS and 04303+2240 have featureless continua and negligible TiO absorption on their spectra. Other stars with strong optical continua have modest to strong TiO absorption bands and must have M-type central stars.

The measured $\mathrm{TiO}$ indices indicate optical veiling in two sources with MMT or Palomar spectra. The class I sources HH32 IRS2 and 04158+2805 lie above the pre-main-sequence locus in Figure 6. In both cases, the M-type absorption features for $\lambda>7400 \AA$ are very strong, which suggests that the $[\mathrm{TiO}]_{1}$ index is 'weak' compared to the $[\mathrm{TiO}]_{2}$ index. Many $\mathrm{T}$ Tauri stars with strong emission lines also have weak $[\mathrm{TiO}]_{1}$ indices. These $\mathrm{T}$ Tauri stars have substantial emission from a blue continuum source which veils optical absorption lines; this veiling increases towards short wavelengths in all cases (see Hartigan et al. 1991). Optical veiling from a hot, $T \sim 10^{4} \mathrm{~K}$, continuum source probably causes the weak $[\mathrm{TiO}]_{1}$ index in HH32 IRS2 and $04158+2805$, but our optical spectra have insufficient signal-to-noise to verify that absorption features at $\lambda<6000 \AA$ are similarly weakened.

We derive spectral types for class I sources and faint $\mathrm{T}$ Tauri stars via comparison with $\mathrm{T}$ Tauri stars of known spectral type. We adopt spectral types for bright $\mathrm{T}$ Tauri stars from KH95 and use FAST spectra to calibrate $[\mathrm{TiO}]_{1}$ and $[\mathrm{TiO}]_{2}$ as a function of spectral type. The measured $\mathrm{TiO}$ indices for class I sources then yield the spectral types listed in Table 1 . We estimate probable errors of $\pm 1-2$ subclasses for the spectral types based on measurement errors of the $\mathrm{TiO}$ indices and the intrinsic uncertainty in assigning spectral types to bright $\mathrm{T}$ Tauri stars.

\subsection{Jet Emission}

As we noted in the introduction, powerful optical and molecular outflows distinguish class I sources from other pre-main-sequence stars in nearby dark clouds. Practically all class I sources have molecular outflows; very few optically visible T Tauri stars are associated with molecular outflows (e.g., Bontemps et al. 1996). In their complete survey of Taurus class I sources, Gómez et al. (1997) show that the frequency of optical jets decreases from $\gtrsim 60 \%$ for class I sources to $\lesssim 10 \%$ for T Tauri stars. Among T Tauri stars, optical jet emission is almost always associated with classical T Tauri stars (CTTS) instead of weak-emission T Tauri stars (WTTS; see Edwards et al. 1987; Hartigan et al. 1995). Near-infrared surveys also indicate more emission line activity among class I sources than CTTS or WTTS (Greene \& Lada 1996a, 1996b). These results suggest that jet emission is correlated with disc accretion and that disc accretion somehow declines from class I sources to T Tauri stars (Edwards et al. 1987; Hartigan et al. 1995; Greene \& Lada 1996b).

To examine these correlations with our spectroscopic data, we divide sources into 
classes based on the ratio of far-infrared to bolometric luminosity, $L_{F I R} / L_{\mathrm{b}}(\mathrm{KH} 95)$. In this system, class I sources have $L_{F I R} / L_{\mathrm{b}} \geq 0.8$, CTTS have $L_{F I R} / L_{\mathrm{b}} \approx 0.1-0.3$, and WTTS sources have $L_{F I R} / L_{\mathrm{b}} \leq 0.1$. We also select 14 sources in Tables $1-2$ with $L_{F I R} / L_{\mathrm{b}} \approx$ 0.3-0.8 as flat-spectrum sources (see also KH95; Greene \& Lada 1996b, 1997). All of these sources have the same median luminosity, $L_{\mathrm{b}} \approx 0.5-0.8 \mathrm{~L}_{\odot}$, except for the flat-spectrum which have $L_{\mathrm{b}} \approx 1.5 \mathrm{~L}_{\odot}(\mathrm{KH} 95)$. For each class, we compute the detection frequency for each of the emission lines listed in Tables 1-2. Figure 7 shows our results for [S II]. The frequency of $[\mathrm{S} \mathrm{II}]$ emission obviously increases with increasing $L_{F I R} / L_{\mathrm{b}}$. We find a similarly striking trend for [N II] emission: practically all class I sources have strong [N II] but only a few CTTS or WTTS have any [N II] emission.

Our detection frequencies for forbidden-line emission are lower limits, because we cannot detect weak emission lines with $\mathrm{EW} \lesssim 0.2-0.4 \AA$ (see Tables $1-2$ ). To estimate the importance of this uncertainty, we compare our results with Hartigan et al. (1995) who derive emission line equivalent widths from echelle spectra. At higher resolution, the detection frequency for [S II] emission increases to $\sim 50 \%$ for CTTS and remains unchanged at $0 \%$ for WTTS. The [N II] emission shows a similar trend but is detected less often than [S II]. Hartigan et al. note, however, that the forbidden emission lines in CTTS consist of high velocity material from the jet and low velocity gas near the disc. The weak, low velocity emission is responsible for the larger [S II] detection frequency among CTTS in the Hartigan et al. sample. Our low resolution spectra do not detect this emission and thus provide a proper estimate for the frequency of high velocity jet emission among CTTS.

To check further the reality of the trend in Figure 7, we perform a simple test. We assume a parent population of $N$ sources with an intrinsic probability, $p$, of [S II] emission. For $n_{j}$ observed sources, the probability of detecting [S II] emission in $k_{j}$ sources is given by the binomial distribution:

$$
p_{o b s}=\frac{n_{j} !}{k_{j} !\left(n_{j}-k_{j}\right) !} p^{k_{j}}(1-p)^{\left(n_{j}-k_{j}\right)}
$$

for $n_{j}<N$. If we require $p_{\text {obs }} \gtrsim 10^{-3}$, the allowed range in $p$ for a single class of pre-main-sequence star is large: $p \lesssim 0.13$ for WTTS, $p \approx 0.03-0.36$ for CTTS, $p \approx$ $0.24-0.93$ for flat-spectrum sources, and $p \approx 0.33-1.00$ for class I sources. However, the probability of realizing the observed frequency of $[\mathrm{S} \mathrm{II}]$ detection for any two classes from a single parent population is extremely small. The probability that the WTTS and CTTS in our sample have the same parent distribution never exceeds $10^{-3}$; it exceeds $10^{-4}$ only for $p=0.04-0.12$. We find no common intrinsic probability for the CTTS and flat spectrum sources or for CTTS and class I sources: $p_{\text {obs }}$ is less than $10^{-4}$ for any value of $p$. The observed frequencies of [S II] emission in class I and flat spectrum sources, however, could be chosen from the same parent population for $p=0.46-0.90$ if $p_{\text {obs }} \geq 10^{-3}$.

We derive similar results for the frequency of [N II] emission. We detect the $\lambda \lambda 6548$, 6584 doublet in $82 \%$ of 11 class I sources, $36 \%$ of 14 flat spectrum sources, $4 \%$ of 46 CTTS, 
and $0 \%$ of 54 WTTS with reliable $L_{F I R} / L_{\mathrm{b}}$. The allowed ranges in the intrinsic probability of [N II] emission are then $p \gtrsim 0.33$ for class I sources, $p \approx 0.07-0.76$ for flat-spectrum sources, $p \lesssim 0.21$ for CTTS, and $p \lesssim 0.12$ for WTTS. The observed detection frequencies allow a single intrinsic probability for [N II] emission between CTTS and WTTS for $p \lesssim$ 0.11 and between class I and flat spectrum sources for $p \approx 0.40-0.71$. Our data do not allow a single [N II] emission probability for all sources. This result is not as strong as for the [S II] lines, because weak [N II] emission is more difficult to detect due to the strong $\mathrm{H} \alpha$ lines in many CTTS.

We conclude that the increasing frequency of forbidden line emission as a function of $L_{F I R} / L_{\mathrm{b}}$ is real. Sources with large $L_{F I R} / L_{\mathrm{b}}$ are much more likely to be associated with forbidden line emission than sources with small $L_{F I R} / L_{\mathrm{b}}$. In addition, our sample is large enough to detect a significant difference in the frequency of [S II] emission between CTTS and WTTS. Previously published data had suggested this difference, but the data were too heterogeneous to make a firm statistical comparison. Our sample is not large enough to detect a difference in the $[\mathrm{N} \mathrm{II}]$ or $[\mathrm{S} \mathrm{II}]$ emission frequency between class I and flat spectrum sources. Adding sources to the optical sample and improved measurements of $L_{F I R} / L_{\mathrm{b}}$ would allow a better discriminant between class I and flat spectrum sources. These improvements require larger ground-based telescopes and new far-IR data from either ISO or SIRTF.

\subsection{The HR Diagram}

Figure 8 shows an HR diagram for Taurus-Auriga pre-main-sequence stars (see KH95). Filled circles plot WTTS and CTTS from KH95円. Crosses indicate the positions of $04158+2805$, HH31 IRS2, and 04489+3042 using our new spectral types. The triangle indicates Haro 6-28 with a revised spectral type (M2) based on FAST spectra. The diamonds denote $04181+2655$ and $04295+2251$ using near-IR spectra from Greene \& Lada (1996b). The relative positions of these two class I sources should be accepted with some caution, because near-IR spectral types for 04489+3042 (K1-K2) and Haro 6-28 (K5-K6) are much earlier than our optical spectral types.

Although the class I sample is small, the positions of class I sources are not especially distinct from the distribution of WTTS and CTTS. Three class I sources straddle the $10^{6}$ yr isochrone from the CMA models of D'Antona \& Mazzitelli (1994). Two other class I sources fall midway between the $10^{5} \mathrm{yr}$ and $10^{6} \mathrm{yr}$ isochrones. All class I sources are within $2 \sigma$ of the $10^{5} \mathrm{yr}$ isochrone and the stellar "birthline" for spherical accretion (Stahler 1983, 1988; Palla \& Stahler 1990, 1993; see also KH95). Only Haro 6-28, however, lies on the

\footnotetext{
${ }^{1}$ We use the KH95 conversion from spectral type to effective temperature for the class I sources.
} 
stellar birthline?

To compare our new data with an alternative to spherical protostellar accretion theory (Stahler 1983, 1988), we consider the evolution of protostars accreting material from a disc. We construct a set of stellar models using the most recent version of the Eggleton evolution program (Eggleton 1971, 1972, 1973). Our models assume an initially uniform composition with abundances of hydrogen $X=0.7$, helium $Y=0.28$, deuterium $X_{\mathrm{D}}=3.5 \times 10^{-5}$, and metals $Z=0.02$ appropriate for the meteoritic mixture determined by Anders \& Grevesse (1989). Pols et al. (1995) describe the equation of state, which includes molecular hydrogen, pressure ionization, and coulomb interactions. The nuclear reaction network includes the pp chain and the CNO cycles. Deuterium burning is explicitly included at temperatures too low for the pp chain. Once the pp chain is active hydrogen burns to $\mathrm{He}^{4}$ with deuterium and $\mathrm{He}^{3}$ in equilibrium. The burning of $\mathrm{He}^{3}$ is not explicitly followed. We use the opacity tables of Iglesias, Rogers \& Wilson (1992) and Alexander \& Ferguson (1994). We adopt an Eddington approximation (Woolley \& Stibbs 1953) for the surface boundary conditions at an optical depth of $\tau=2 / 3$. Low-temperature atmospheres, in which convection extends out as far as $\tau \approx 0.01$ (Baraffe et al. 1995), are not modeled completely. However the effect on observable quantities is not significant (see Kroupa \& Tout 1998).

In these calculations, the initial protostar is a fully convective $0.1 M_{\odot}$ object with a radius of $3 R_{\odot}$ and an effective temperature of $10^{3.43} \mathrm{~K}$. This starting point lies just off the right boundary of Figure 8. We add accreted material to the surface with the initial composition and with the same state (entropy, temperature, etc.) as the surface. In this approximation, most of the stellar surface is free to radiate normally with the boundary conditions described above. These boundary conditions are a compromise, because we are modeling a two dimensional process with a one dimensional evolution code.

The solid lines in Figure 8 indicate two accreting protostellar tracks using the Eggleton code. The thinner line accretes at $10^{-6} \mathrm{M}_{\odot} \mathrm{yr}^{-1}$; the thick line accretes at $10^{-5} \mathrm{M}_{\odot} \mathrm{yr}^{-1}$. Initially, both stars contract at fairly constant luminosity and move to the left in the HR diagram. This contraction continues until deuterium ignites at their centers. The tracks then turn upwards to follow a deuterium burning sequence. Both protostars are fully convective, so newly accreted material replenishes central deuterium. These protostars thus remain on the deuterium burning track until either accretion ceases or the rate of deuterium replenishment becomes insufficient for burning to continue to support the star. The latter occurs first, temporally, for the $10^{-5} \mathrm{M}_{\odot} \mathrm{yr}^{-1}$ model at $T_{\text {eff }}=10^{3.62} \mathrm{~K}$ when it has reached a mass of $0.686 M_{\odot}$. The $10^{-6} \mathrm{M}_{\odot} \mathrm{yr}^{-1}$ model drops below the deuterium sequence at $T_{\text {eff }}=10^{3.58} \mathrm{~K}$ at a mass of $0.456 M_{\odot}$. In both cases, we continue accretion until the total mass reaches $0.96 M_{\odot}$. During the protostellar evolution, the stars lie close to the Hayashi tracks appropriate to their instantaneous mass. If accretion ceases at any time, the star will

\footnotetext{
${ }^{2}$ We suspect that Haro 6-28 may be a close binary similar to GV Tau (Haro 6-10), because their spectral energy distributions are similar. Both GV Tau and Haro 6-28 are 2-3 mag brighter in the optical than other class I sources in the sample.
} 
shrink down to the main-sequence along a normal pre-main-sequence track and will ignite and burn deuterium on the way if it has not already done so.

In our accretion models, the deuterium burning sequence defines a stellar birthline in the HR diagram. Previous calculations find similar results. Stahler (1983, 1988; see also Palla \& Stahler 1990, 1993) first identified the birthline for spherical accretion and showed that this locus provides a good upper envelope for observations of young stars in nearby molecular clouds. Mercer-Smith et al. (1984) published the first HR diagram track for a disc-accreting protostar using a code similar in spirit to the Eggleton code. Hartmann et al. (1997) later derived a birthline for disc accretion from semi-analytic calculations. Our deuterium burning sequence lies close to Stahler's $(1983,1988)$ birthline for $\log T_{\text {eff }} \gtrsim$ 3.55 and falls $\delta \log L \approx 0.1-0.2$ below the birthline for $\log T_{\text {eff }} \lesssim 3.55$. The displacement reflects differences in the starting conditions and outer boundary condition. The birthlines converge at large $T_{\text {eff }}$, because the boundary conditions become less important as the stellar luminosity increases (see also Palla \& Stahler 1990, 1993).

The location of the stellar birthline in our models is sensitive to the adopted deuterium abundance. This behavior follows from the explicit dependence of the stellar luminosity on the rate of deuterium burning (see, for example, Stahler 1988; Hartmann et al. 1997). Our deuterium burning sequence shifts by $\delta \log L \approx-0.13$ for a factor of two reduction in $\mathrm{X}_{\mathrm{D}}$. Our birthline then roughly coincides with that of Hartmann et al. (1997) for $\mathrm{X}_{\mathrm{D}}=1.75 \times 10^{-5}$.

With only six class I sources in our HR diagram, it is difficult for the data to favor convincingly any theoretical calculation. The $2 \sigma$ error bars are consistent with all of the tracks, even without considering uncertainties in the model input parameters. The data lie closer to the disc-accretion birthline of Hartmann et al. (1997) and our deuterium burning sequence than either the birthline for spherical accretion or the accretion track of Mercer-Smith et al. (1984). In all cases, changing model input parameters - such as the deuterium abundance - would allow a better match between data and the models. More rigorous comparisons thus await observational estimates of unknown quantities such as the deuterium abundance and a larger sample of class I sources with reliable spectral types and luminosities.

We conclude this section with several points about the comparison of observations with model tracks. First, the distribution of class I sources about any birthline should be uniform, if the range in initial conditions is small. This dispersion should be comparable to the observational errors. In our case, 5 out of 6 class I sources fall $1-2 \sigma$ below the birthline for spherical accretion (Stahler 1983, 1988) and the Mercer-Smith et al. (1984) accretion track. The data are somewhat more consistent with our deuterium burning sequence and the Hartmann et al. (1997) birthline for an accretion rate of $10^{-5} \mathrm{M}_{\odot} \mathrm{yr}^{-1}$. A larger sample of class I sources should distinguish between models.

Second, it is important to compare the stellar component of the protostellar luminosity with the predictions of model tracks. The observed class I luminosities are the total 
luminosity and have not been corrected for the unknown amount of accretion luminosity. In most of the CTTS shown in Figure 8, the accretion luminosity is a small fraction $10 \%$ to $30 \%$ - of the stellar luminosity plotted in the figure (see Hartigan et al. 1991, 1995; Gullbring et al. 1998). However, the accretion luminosity is roughly comparable to the stellar luminosity in the continuum + emission sources and is $\sim 100$ times the stellar luminosity in FU Ori systems like L1551 IRS5 (Hartigan et al. 1991; Hartmann \& Kenyon 1996). We expect a small accretion contribution for class I sources with optical spectra similar to most CTTS, although the accretion luminosity in L1489 IRS may be large (see also Greene \& Lada 1996b). The arrow in Fig. 8 indicates the displacement in $\log T_{\text {eff }}$ for a $\delta \log L=-0.1$ change in the bolometric luminosity. This change moves the data closer to both the birthline for spherical accretion and our deuterium sequence and illustrates the difficulty in comparing models with current data.

Third, knowledge of the deuterium abundance, and to a lesser extent the lithium abundance, is also necessary to compare observations with model predictions. The mean deuterium abundance for stars in a molecular cloud sets the location of the birthline in the HR diagram; any star-to-star scatter in the abundance spreads the birthline vertically in the HR diagram. To our knowledge, the deuterium abundance has not been measured in any pre-main-sequence star. Recent measurements indicate a factor of 3-5 scatter in the lithium abundance among nearby molecular clouds (e.g., King 1993; Lee et al. 1994; Duncan \& Rebull 1996) that could be due actual abundance differences between stars (see Lee et al. 1994) or differences in the analysis procedures (see Duncan \& Rebull 1996 and references therein). Observations of older open clusters may also indicate considerable star-to-star differences in the rate of lithium depletion among stars with the same mass (e.g., Randich et al. 1997 and references therein). Similar spreads in the deuterium burning rate, due perhaps to star-to-star variations in accretion rate, further complicates the comparison of observations with model tracks. The sample of protostars is not currently large enough to worry about abundance variations, but the uncertainties will become more important as sample sizes increase.

\section{DISCUSSION and SUMMARY}

In the previous sections, we have described the first optical spectroscopic survey of class I, embedded sources in a single molecular cloud. We supplemented these data with high quality optical spectra of a representative sample of older and optically brighter $\mathrm{T}$ Tauri stars. The combined set of spectra show that the optical spectra of class I sources qualitatively resemble the optical spectra of $\mathrm{T}$ Tauri stars. Our analysis further reveals common physical properties and substantial differences between class I sources and T Tauri stars, as summarized below.

Our data provide the first indication that the distribution of stellar spectral types

among class I sources may not be very different from that of WTTS and CTTS. Of the five 
class I sources with strong optical continua, one (L1489 IRS) is a continuum + emission source, three are M-type stars, and another $(04264+2433)$ may have an M-type central star. To the best of our knowledge, these are the first low mass protostars with measured optical spectral types. This sample is too small for a meaningful comparison with the distribution of spectral types among more evolved pre-main-sequence stars in the cloud. We note, however, that the median spectral type for WTTS and CTTS is K7-M0 and that the frequency of continuum + emission sources is $\sim 5 \%-10 \%$ (KH95).

Published observations indicate other similarities between class I sources and older pre-main-sequence stars in Taurus-Auriga. First, class I sources have the same intrinsic near-IR colors as do CTTS. Whitney et al. (1997) show that the observed near-IR colors of class I sources can be modeled as a CTTS surrounded by an infalling envelope with an optical extinction, $A_{V} \approx 30-60 \mathrm{mag}$. This analysis leads to the conclusion that the radiation from the star and inner disc of a class I source is similar to that of a T Tauri star (see also Greene \& Lada 1996b; Calvet et al. 1997 reach a different conclusion). Second, the bolometric luminosity distributions of class I sources, CTTS, and WTTS are indistinguishable (KH95). All three groups of pre-main-sequence stars have median luminosities of $L_{\mathrm{b}} \approx 0.5-0.8 \mathrm{~L}_{\odot}$. This unexpected result is supported by the positions of class I sources in the HR diagram. Our data show that class I sources have luminosities and effective temperatures very similar to those of CTTS and WTTS in the cloud. These conclusions are surprising, because a class I source should have a larger luminosity once it has accreted nearly all of its final mass, and this luminosity should decline with time as the star approaches the main sequence (see, for example, Stahler 1983, 1988; Palla \& Stahler 1993; Hartmann et al. 1997; Fig. 8). The current sample, however, is too small to test stellar models in detail. The errors in luminosity and effective temperature are also too large. Observations with the next generation of ground-based telescopes will undoubtedly expand the sample, reduce the errors, and provide better tests of protostellar accretion theory.

One feature that distinguishes class I sources is their strong forbidden-line emission. As a group, class I sources are much more likely to have forbidden-line emission than CTTS or WTTS (Fig. 7). This result confirms previous conclusions from imaging data (e.g., Gómez et al. 1997) and indicates that class I sources are more likely to drive outflows than CTTS or WTTS (see also Bontemps et al. 1996; Moriarty-Schieven et al. 1992, 1994). The equivalent widths of the forbidden lines are also larger in class I sources than in CTTS or WTTS. Although some large equivalent widths may be due to very weak optical continua, the [S II] equivalent widths in HH31 IRS2 - a class I source with a prominent TiO absorption band - are larger than observed in any CTTS in our sample (see Tables 1-2). Deeper optical spectra of our sample and other class I sources would clarify this point.

Our sample is not large enough to test whether class I sources also have more prominent permitted emission lines than CTTS. The median $\mathrm{H} \alpha$ equivalent width for class I sources, $\sim 90 \AA$, is much larger than the median equivalent width for CTTS, $\sim 30-40 \AA$. This difference is roughly what we expect if class I sources have larger continuum veiling than 
CTTS (Casali \& Eiroa 1996; Greene \& Lada 1997) and if the H $\alpha$ equivalent width correlates with veiling (Hartigan et al. 1995 and references therein). However, the frequency of He I $\lambda \lambda 5876,6678$ emission among class I sources is roughly comparable to that among CTTS. We measure a He I emission frequency of $50 \%$ among 6 class I sources with reasonable signal-to-noise at $6000 \AA, 57 \%$ among 14 flat-spectrum sources, and $65 \%$ among 46 class II sources. For both emission lines, the class I sample is probably biased against small equivalent widths, because class I sources without emission lines are probably fainter than sources with emission lines. A deeper survey with a larger telescope could enlarge the sample of class I sources with high quality optical spectra. These data would provide a good test for differences in the distribution of $\mathrm{H} \alpha$ equivalent widths between class I sources and CTTS.

These results fit into the general picture of Taurus-Auriga class I sources developed in KH95 and in Kenyon et al. (1990). In this picture, class I sources are envelopes of gas and dust falling into the central star-disc system at rates of a few $\times 10^{-6} \mathrm{M}_{\odot} \mathrm{yr}^{-1}$ (see also Adams et al. 1987; Kenyon et al. 1993a, 1993b; Whitney et al. 1997). Bell \& Lin (1994) show that the stable accretion rate through the disc onto the central star is either very low $-\lesssim$ a few $\times 10^{-7} \mathrm{M}_{\odot} \mathrm{yr}^{-1}-$ or very high $-\gtrsim$ a few $\times 10^{-5} \mathrm{M}_{\odot} \mathrm{yr}^{-1}-$ compared to the infall rate. The disc spends most of its time in the low accretion rate state; the disc mass then slowly increases with time until it reaches a critical level and evolves to the high accretion rate state. This model explains the low observed luminosities of nearly all class I sources as well as the occasional high luminosity of a source such as L1551 IRS5.

Models with time-dependent disc accretion also qualitatively account for the evolution of forbidden and permitted emission lines in pre-main-sequence stars. We expect the time-averaged accretion rate through the disc to decline as the envelope disperses. If the $\mathrm{H} \alpha$ and other permitted emission lines of class I sources form in the accretion region of the inner disc as in CTTS, then the median $\mathrm{H} \alpha$ equivalent width should decline as a pre-main sequence star evolves from a class I source into a CTTS and then into a WTTS. Most models for jet formation link the mass loss rate in the jet to the mass accretion rate through the disc (see, for example, Cabrit et al. 1990; Najita \& Shu 1994; Shu et al. 1994a, 1994b), so we expect forbidden emission to decline as well. Explaining the observations of emission line equivalent widths with a quantitative model of a dispersing envelope and evolving disc, however, is currently beyond our reach.

Finally, our results further demonstrate the advantages of optical spectra. Recent surveys of larger samples of class I sources using near-IR spectroscopy have yielded only two spectral types each in Taurus-Auriga (Casali \& Eiroa 1996; Greene \& Lada 1996b) and $\rho$ Oph (Greene \& Lada 1996b, 1997). Casali \& Eiroa (1996; see also Casali \& Matthews 1992; Greene \& Lada 1996b, 1997) conclude that continuum emission from dust in a circumstellar disc or envelope veils photospheric absorption features on near-IR spectra of class I sources. Preliminary results further suggest that this veiling is larger in class I sources than in CTTS or WTTS (Casali \& Eiroa 1996; Greene \& Lada 1996b, 1997). Dust emission is much weaker relative to a normal stellar photosphere at shorter wavelengths, $\lesssim 1 \mu \mathrm{m}$, so optical 
spectra may yet provide the best measure of spectral types in class I sources.

We thank the staffs of the MMT, Palomar, and Whipple Observatories for assistance with our observations. Fred Chaffee kindly acquired several spectra of the class II sources listed in Table 1. Susan Tokarz reduced the FAST spectra and graciously assisted with the reduction of the MMT and Palomar spectra. We also thank M. Geller, M. Gómez, C. Lada, A. Mahdavi, and B. Whitney for advice and comments. The suggestions of an anonymous referee improved our presentation. Observations at the Palomar Observatory were made as part of a continuing collaborative agreement between Palomar Observatory and the Jet Propulsion Laboratory. Portions of this research were supported by the National Aeronautics and Space Administration through grant NAGW-2919 and by the Space Telescope Science Institute through grant GO-06132.01-94A. C.A.T. thanks the Royal Society and the Hungarian Academy of Sciences for an exchange fellowship during the majority of his contribution to this project. 


\section{REFERENCES}

Adams, F. C. 1990, ApJ, 363, 578

Adams, F. C., Lada, C. J., \& Shu, F. H. 1987, ApJ, 312, 788

Alexander, D. R., \& Ferguson, J. W. 1994, ApJ, 437, 879

Anders E., \& Grevesse N., 1989, Geochim. Cosmochim. Acta, 53, 197

André, P., Ward-Thompson, D., \& Barsony, M. 1993, ApJ, 406, 122

Baraffe, I., Chabrier, G., Allard, F., \& Hauschildt, P. H. 1995, ApJL, 446, L35

Basri, G., \& Bertout, C. 1993, in Protostars and Planets III, eds. E. H. Levy \& J. I. Lunine, Tucson, Univ of Arizona, p. 543

Böhm, K. H., \& Solf, J. 1990, ApJ, 348, 297

Bontemps, S., André, P., Terebey, S., \& Cabrit, S. 1996, A\&A, 311, 858

Cabrit, S., Edwards, S., Strom, S. E., Strom, K. M. 1990, ApJ, 354, 687

Calvet, N., Hartmann, L., \& Strom, S. E. 1997, ApJ, 481, 912

Casali, M. M., \& Eiroa, C. 1996, A\&A, 306, 427

Casali, M. M., \& Matthews, H. E. 1992, MNRAS, 258, 399

Cohen, M., \& Kuhi, L. V. 1979, ApJS, 41, 743

D’Antona, F., \& Mazzitelli, I. 1994, ApJS, 90, 467

Dopita, M. A., Schwartz, R. D., \& Evans, I. 1982, ApJL, 263, L73

Duncan, D. K. \& Rebull, L. M. 1996, PASP, 108, 738

Edwards, S., Cabrit, S., Strom, S. E., Heyer, I., Strom, K. M., \& Anderson, E. 1987, ApJ, 321,473

Eggleton, P. P. 1971, MNRAS, 151, 351

Eggleton, P. P. 1972, MNRAS, 156, 361

Eggleton, P. P. 1973, MNRAS, 163, 279

Eiroa, C., Miranda, L. F., Anglada, G., Estalella, R., \& Torrelles, J. M. 1994, A\&A, 283, 973

Fabricant, D. G., Cheimets, P., Caldwell, N., \& Geary, J. 1998, PASP, 110, 79 
Fletcher, A. B., \& Stahler, S. W. 1994a, ApJ, 435, 313

Fletcher, A. B., \& Stahler, S. W. 1994b, ApJ, 435, 329

Gómez, M., Whitney, B. A., \& Kenyon, S. J. 1997, AJ, 114, 1138

Goodrich, R. W. 1986, AJ, 92, 885

Graham, J. A., \& Rubin, V. C. 1992, PASP, 104, 730

Greene, T. P., \& Lada, C. J. 1996a, ApJ, 461, 345

Greene, T. P., \& Lada, C. J. 1996b, AJ, 112, 2184

Greene, T. P., \& Lada, C. J. 1997, AJ, 114, 114, 2157

Greene, T. P., Wilking, B. A., André, P., Young, E. T., \& Lada, C. J. 1994, ApJ, 434, 614

Gullbring, E., Hartmann, L., Briceno, C., \& Calver, N. 1998, ApJ, 492, 323

Hamman, F., \& Persson, S. E. 1992, ApJS, 82, 247

Hartigan, P., Edwards, S., \& Ghandour, L. 1995, ApJ, 452, 736

Hartigan, P., Hartmann, L., Kenyon, S. J., Hewett, R., \& Stauffer, J. 1989, ApJS, 70, 899

Hartigan, P., Kenyon, S. J., Hartmann, L., Strom, S. E., Edwards, S., Welty, A. D., \& Stauffer, J. 1991, ApJ, 382, 617

Hartmann, L., \& Kenyon, S. J. 1996, ARA\&A, 34, 207

Hartmann, L., Cassen, P., \& Kenyon, S. J. 1997, ApJ, 475, 770

Hogerheijde, M. R., van Dishoeck, E. F., Blake, G. A., \& van Langevelde, H. J. 1997, ApJ, 489, 293

Iglesias, C. A., Rogers, F. J., \& Wilson, B. G. 1992, ApJ, 397, 717 (OPAL)

Kenyon, S. J., Calvet, N., \& Hartmann, L. 1993a, ApJ, 414, 676 (Paper I)

Kenyon, S. J., \& Fernández-Castro, T. 1987, AJ, 93, 938

Kenyon, S. J., \& Hartmann, L. 1995, ApJS, 101, 117 (KH95)

Kenyon, S. J., Hartmann, L. W., Strom, K. M., \& Strom, S. E. 1990, AJ, 99, 869

Kenyon, S. J., \& Hartmann, L. 1990, ApJ, 349, 197

Kenyon, S. J., Yi, I., \& Hartmann, L. 1996, ApJ, 462, 439 
Kenyon, S. J., Whitney, B., Gómez, M., \& Hartmann, L. 1993b, ApJ, 414, 773

King, J. R. 1993, AJ, 105, 1087

Kroupa, P., \& Tout, C. A., 1998, MNRAS, in press

Lada, C. J. 1987, in Star Forming Regions, edited by M. Peimbert \& J. Jugaka, Kluwer, Dordrecht, p. 1

Lee, C. W., Martin, E. L., \& Mathieu, R. D. 1994, AJ, 108, 1445

Martin, S. C. 1997, ApJL, 478, L33

Mercer-Smith, J. A., Cameron, A. G. W., \& Epstein, R. I. 1984, ApJ, 279, 363

Moriarty-Schieven, G. H., Wannier, P. G., Mangum, J. G., Tamura, M., \& Olmstead, V. K. 1995b, ApJ, 455, 190

Moriarty-Schieven, G. H., Wannier, P. G., Tamura, M., Keene, J. B. 1992, ApJ, 400, 260

Myers, P. C., Adams, F. C., Chen, H., \& Schaff, E. 1998, ApJ, in press

Myers, P. C., Fuller, G. A., Mathieu, R. D., Beichman, C. A., Benson, P. J., Schild, R. E., \& Emerson, J. P. 1987, ApJ, 319, 340

Najita, J. R., \& Shu, F. H. 1994, ApJ, 429, 808

O’Connell, R. W. 1973, AJ, 78, 1074

Palla, F., \& Stahler, S. W. 1990, ApJL, 360, L47

Palla, F., \& Stahler, S. W. 1993, ApJ, 418, 414

Pols, O. R., Tout, C. A., Eggleton, P. P., \& Han, Z. 1995, MNRAS, 274, 964

Raga, A. C., Böhm, K.-H., \& Canto, J. 1996, Rev Mex A\&A, 32, 161

Randich, S., Aharpour, N., Pallavicini, R., Prosser, C. F., \& Stauffer, J. R. 1997, A\&A, 323,86

Reipurth, B., \& Heathcote, S. 1991, A\&A, 246, 511

Saraceno, O., André, P., Ceccarelli, C., Griffin, M., \& Molinari, S. 1996, A\&A, 309, 827

Shu, F. H., Najita, J., Ostriker, E., Wilkin, F., Ruden, S. P., \& Lizano, S. 1994a, ApJ, 429, 781

Shu, F. H., Najita, J., Ruden, S. P., \& Lizano, S. 1994b, ApJ, 429, 797

Stahler, S. W. 1983, ApJ, 274, 822 
Stahler, S. W. 1988, ApJ, 332, 804

Stocke, J. T., Hartigan P. M., Strom, S. E., Strom, K. M., Anderson, E. R., Hartmann, L. W., \& Kenyon, S. J. 1988, ApJS, 68, 229

Tamura, M., Gatley, I., Joyce, R. R., Ueno, M., Suto, H., \& Sekiguchi, M. 1991b, ApJ, 378, 611

Tamura, M., Gatley, I., Waller, W. \& Werner, M. W. 1991a, ApJ, 374, L25

Terebey, S., Chandler, C. J., \& André, P. 1993, ApJ, 414, 759

Walter, F. M., Brown, A., Linsky, J. L., Rydgren, A. E., Vrba, F., Roth, M., Carrasco, L., Chugainov, P. F., Shakovskaya, N. I., \& Imhoff, C. L. 1987, ApJ, 314, 297

Whitney, B. A., Kenyon, S. J., \& Gómez, M. 1997, ApJ, 485, 703

Wilking, B. A., Lada, C. J., \& Young, E. T. 1989, ApJ, 340, 823

Woolley, R. v. d. R., \& Stibbs, D. W. N. 1953. The Outer Layers of a Star. Clarendon Press, Oxford 
Table 1. Spectral types and equivalent widths from MMT and Palomar spectra

\begin{tabular}{|c|c|c|c|c|c|c|c|c|c|}
\hline Source Name & SED & $L_{F I R} / L_{\mathrm{b}}$ & ST & [O I] 6300 & [O I] 6363 & $\mathrm{H} \alpha$ & [N II] 6584 & [S II] 6717 & {$[\mathrm{~S}$ II $] 6730$} \\
\hline L1489 IRS & $\mathrm{I}$ & 0.85 & $\mathrm{Ce}$ & $\cdots$ & $\cdots$ & 55.5 & 1.5 & 2.0 & $\cdots$ \\
\hline L1489 IRS & I & 0.85 & $\mathrm{Ce}$ & $\cdots$ & $\cdots$ & 56.0 & 1.4 & 1.3 & 1.4 \\
\hline $04108+2805$ & II & $\cdots$ & M0 & $\cdots$ & $\cdots$ & 37.0 & $\cdots$ & $\cdots$ & $\cdots$ \\
\hline $04113+2758$ & II & $<0.77$ & M2 & $\cdots$ & $\cdots$ & 49.0 & $\cdots$ & $\ldots$ & $\cdots$ \\
\hline $\mathrm{CoKu} \mathrm{Tau/1}$ & II & $\cdots$ & M0 & 71.5 & 23.0 & 110.5 & 33.5 & 20.0 & 31.0 \\
\hline $04158+2805$ & I & $<0.93$ & M3 & 91.0 & 35.5 & 326.0 & 24.4 & 13.5 & 18.0 \\
\hline $04216+2603$ & II & $<0.48$ & M0 & $\cdots$ & $\cdots$ & 26.5 & $\cdots$ & $\cdots$ & $\cdots$ \\
\hline $04239+2436$ & I & 0.91 & $\ldots$ & $\ldots$ & $\ldots$ & $>200.0$ & $>100.0$ & $>100$ & $>200.0$ \\
\hline HH31 IRS2 & $\mathrm{I}$ & 0.92 & M2 & $\cdots$ & $\cdots$ & 86.0 & 13.0 & 8.0 & 10.0 \\
\hline HH31 IRS2 & $\mathrm{I}$ & 0.92 & M2 & $\cdots$ & $\cdots$ & 95.0 & 7.5 & 6.5 & 9.0 \\
\hline $04260+2642$ & $\mathrm{I}$ & 0.89 & $\cdots$ & 121.0 & 38.0 & 125.0 & 14.5 & 13.5 & 24.5 \\
\hline $04264+2433$ & $\mathrm{I}$ & 0.95 & M0? & 23.0 & 7.0 & 78.5 & 10.5 & 7.0 & 10.0 \\
\hline L1551 IRS5 & I & 0.98 & G-K & 41.0 & 13.0 & 83.0 & 32.0 & 40.0 & 52.0 \\
\hline $\mathrm{LkH} \alpha 358$ & II & $<0.76$ & M5-M6 & $\ldots$ & $\ldots$ & 86.5 & $\ldots$ & $\cdots$ & $\ldots$ \\
\hline HH30 IRS & I/II & $\cdots$ & M0? & 110.0 & 35.0 & 185.0 & 30.5 & 47.0 & 77.5 \\
\hline $04295+2251$ & I & 0.80 & $\cdots$ & $\cdots$ & $\cdots$ & 65.5 & $\cdots$ & $\cdots$ & $\cdots$ \\
\hline $04303+2240$ & $\mathrm{I}$ & $<0.18$ & $\mathrm{Ce}$ & 20.0 & 7.5 & 122.0 & 16.5 & $\ldots$ & $\cdots$ \\
\hline $04368+2557$ & I & 1.00 & . & $\ldots$ & $\cdots$ & $>200.0$ & $>100.0$ & $>100$ & $>200.0$ \\
\hline $04385+2550$ & II & 0.72 & M0 & $\ldots$ & $\ldots$ & 20.0 & 3.0 & 2.5 & 3.5 \\
\hline $04489+3042$ & I & 0.83 & M2 & 164.0 & 22.5 & 11.0 & 24.5 & 7.0 & 11.0 \\
\hline
\end{tabular}

Notes for Table 1:

1. Three class I sources have detectable He I $\lambda \lambda 5876,6678$ emission with EW $\approx 2-3 \AA$ : L1489 IRS, HH31 IRS2, and $04264+2433$. Two class II sources have comparably strong He I emission: $04216+2603$ and $04303+2240$.

2. L1489 IRS is also known as $04016+2610$.

3. $04185+2805$ is class II in KH95. Our optical spectra - together with unpublished optical and near-IR photometry indicate that this object is a class I source.

4. L1551 IRS5 is also known as $04287+1801$.

5. HH31 IRS2 is also known as $04248+2612$. 
Table 2. Emission line equivalent widths from FAST spectra

\begin{tabular}{|c|c|c|c|c|c|c|}
\hline Source Name & SED & $\overline{L_{F I R} / L_{\mathrm{b}}}$ & $\overline{\mathrm{H} \alpha}$ & [N II] 6584 & He I 6678 & [S II] $6717+6730$ \\
\hline HBC 351 & $\overline{\text { III }}$ & 0.05 & 2.8 & $\cdots$ & $\cdots$ & $\cdots$ \\
\hline HBC 352 & III & $<0.03$ & -2.2 & $\ldots$ & $\ldots$ & $\ldots$ \\
\hline HBC 353 & III & $<0.04$ & -2.1 & $\cdots$ & $\cdots$ & $\cdots$ \\
\hline HBC 354 & III & 0.14 & -1.2 & $\cdots$ & $\cdots$ & $\cdots$ \\
\hline HBC 355 & III & 0.07 & -1.2 & $\cdots$ & $\ldots$ & $\ldots$ \\
\hline HBC 356 & III & 0.06 & 0.8 & $\ldots$ & $\ldots$ & $\ldots$ \\
\hline HBC 357 & III & 0.10 & 1.1 & $\ldots$ & .. & $\ldots$ \\
\hline HBC 358 & III & 0.04 & 10.0 & $\cdots$ & $\cdots$ & $\cdots$ \\
\hline HBC 359 & III & 0.04 & 5.4 & $\ldots$ & $\ldots$ & $\ldots$ \\
\hline HBC 360 & III & $<0.20$ & 6.7 & $\cdots$ & $\cdots$ & $\cdots$ \\
\hline HBC 361 & III & $<0.20$ & 6.4 & $\cdots$ & $\cdots$ & $\cdots$ \\
\hline HBC 362 & III & $<0.15$ & 6.6 & $\cdots$ & $\ldots$ & $\ldots$ \\
\hline $\mathrm{LkCa} 1$ & III & $<0.05$ & 3.4 & $\cdots$ & $\cdots$ & $\cdots$ \\
\hline Anon 1 & III & $<0.02$ & 2.5 & $\ldots$ & $\ldots$ & $\cdots$ \\
\hline $04108+2910$ & II & $<0.43$ & 37.5 & $\cdots$ & . & 2.0 \\
\hline V773 Tau & II & 0.10 & 2.8 & $\ldots$ & ... & $\cdots$ \\
\hline FM Tau & II & 0.19 & 101.0 & $\cdots$ & 1.6 & $\cdots$ \\
\hline FN Tau & II & 0.31 & 16.0 & $\cdots$ & $\cdots$ & $\cdots$ \\
\hline CW Tau & II & 0.23 & 140.0 & $\cdots$ & 1.2 & 1.1 \\
\hline CIDA 1 & II & $<0.18$ & 149.0 & $\cdots$ & $\cdots$ & $\cdots$ \\
\hline $\mathrm{LkCa} 3$ & III & 0.01 & 2.7 & $\cdots$ & $\cdots$ & $\cdots$ \\
\hline FP Tau & III & 0.13 & 27.0 & 0.7 & $\ldots$ & $\cdots$ \\
\hline FO Tau & II & 0.15 & 116.0 & $\cdots$ & 1.5 & $\cdots$ \\
\hline CX Tau & II & 0.17 & 18.0 & $\cdots$ & . & $\cdots$ \\
\hline CIDA 2 & III & $<0.09$ & 6.8 & $\ldots$ & . & $\cdots$ \\
\hline $\mathrm{LkCa} 4$ & III & $<0.02$ & 3.2 & $\cdots$ & . & $\ldots$ \\
\hline CY Tau & II & 0.10 & 63.0 & $\ldots$ & 1.7 & $\cdots$ \\
\hline $\mathrm{LkCa} 5$ & III & 0.16 & 3.8 & $\cdots$ & $\cdots$ & $\cdots$ \\
\hline CIDA 3 & II & $<0.14$ & 10.5 & $\ldots$ & $\ldots$ & $\ldots$ \\
\hline V410 Tau & III & 0.01 & 2.3 & $\ldots$ & $\ldots$ & $\ldots$ \\
\hline DD Tau & II & 0.40 & 177.5 & $\cdots$ & 3.9 & 2.2 \\
\hline CZ Tau & II & 0.29 & 6.7 & $\ldots$ & $\ldots$ & $\ldots$ \\
\hline HBC 372 & III & $<0.15$ & -1.2 & $\cdots$ & . & $\ldots$ \\
\hline V892 Tau & II & 0.58 & 6.6 & $\cdots$ & . & $\cdots$ \\
\hline Hubble 4 & III & $\ldots$ & 3.9 & $\ldots$ & . & $\cdots$ \\
\hline HBC 376 & III & $<0.05$ & 1.9 & $\ldots$ & .. & $\ldots$ \\
\hline FQ Tau & II & 0.15 & 81.0 & $\ldots$ & 3.0 & $\cdots$ \\
\hline BP Tau & II & 0.10 & 92.0 & $\cdots$ & 1.0 & $\cdots$ \\
\hline V819 Tau & III & 0.03 & 3.2 & $\ldots$ & $\ldots$ & $\ldots$ \\
\hline LkCa 7 & III & $<0.02$ & 3.9 & $\cdots$ & $\cdots$ & $\cdots$ \\
\hline J2-157 & III & $<0.50$ & 15.5 & $\cdots$ & $\cdots$ & $\cdots$ \\
\hline DE Tau & II & 0.11 & 59.0 & $\ldots$ & . & $\cdots$ \\
\hline
\end{tabular}


Table 2. Continued

\begin{tabular}{|c|c|c|c|c|c|c|}
\hline Source Name & SED & $\overline{L_{F I R} / L_{\mathrm{b}}}$ & $\overline{\mathrm{H} \alpha}$ & [N II] 6584 & "He I 6678 & [S II] $6717+6730$ \\
\hline RY Tau & II & 0.25 & 13.0 & $\cdots$ & $\cdots$ & \\
\hline HD 283572 & III & $<0.02$ & -1.6 & $\ldots$ & $\ldots$ & .. \\
\hline FS Tau & II & 0.50 & 76.0 & 5.5 & 1.8 & 8.9 \\
\hline T Tau & II & 0.20 & 40.5 & $\cdots$ & 1.7 & 1.7 \\
\hline IP Tau & II & 0.16 & 10.5 & $\ldots$ & $\ldots$ & $\ldots$ \\
\hline $\mathrm{J} 1-4872$ & III & $<0.04$ & 2.9 & $\ldots$ & $\cdots$ & $\ldots$ \\
\hline FV Tau & II & 0.16 & 20.0 & $\ldots$ & $\ldots$ & $\ldots$ \\
\hline DF Tau & II & 0.08 & 54.5 & $\ldots$ & 1.7 & $\ldots$ \\
\hline DG Tau & II & 0.60 & 125.0 & 0.7 & 1.0 & 2.4 \\
\hline HBC 388 & III & 0.02 & -1.6 & $\ldots$ & $\ldots$ & .. \\
\hline DH Tau & II & 0.12 & 38.5 & $\cdots$ & 1.4 & 0.6 \\
\hline DI Tau & II & 0.06 & 2.0 & $\cdots$ & $\cdots$ & $\cdots$ \\
\hline IQ Tau & II & 0.12 & 7.7 & $\cdots$ & $\cdots$ & $\cdots$ \\
\hline FX Tau & II & 0.16 & 14.5 & $\ldots$ & $\ldots$ & $\cdots$ \\
\hline DK Tau & II & 0.18 & 49.5 & $\ldots$ & 1.0 & 1.0 \\
\hline ZZ Tau & III & 0.07 & 14.0 & $\cdots$ & $\cdots$ & $\cdots$ \\
\hline JH 56 & III & $<0.06$ & 2.2 & $\ldots$ & $\ldots$ & $\ldots$ \\
\hline V927 Tau & III & $<0.05$ & 10.0 & $\cdots$ & $\cdots$ & $\cdots$ \\
\hline HBC 392 & III & 0.06 & 1.1 & $\ldots$ & $\ldots$ & $\ldots$ \\
\hline HL Tau & II & 0.77 & 48.0 & $\cdots$ & $\cdots$ & 3.6 \\
\hline XZ Tau & II & 0.61 & 175.0 & $\ldots$ & 1.4 & 2.2 \\
\hline HK Tau & I/II & 0.27 & 53.5 & $\ldots$ & 0.4 & $\ldots$ \\
\hline V710 Tau A & II & 0.08 & 33.5 & $\ldots$ & $\ldots$ & $\ldots$ \\
\hline V710 Tau B & II & 0.14 & 4.3 & $\ldots$ & $\ldots$ & $\ldots$ \\
\hline L1551-51 & III & $<0.04$ & 1.5 & $\cdots$ & $\cdots$ & $\cdots$ \\
\hline V928 Tau & III & $<0.04$ & 1.8 & $\ldots$ & $\ldots$ & $\ldots$ \\
\hline V827 Tau & III & $<0.04$ & 4.4 & $\cdots$ & $\cdots$ & $\cdots$ \\
\hline V826 Tau & III & 0.02 & 3.5 & $\cdots$ & $\cdots$ & $\cdots$ \\
\hline FY Tau & II & 0.12 & 73.0 & $\ldots$ & 0.2 & $\ldots$ \\
\hline FZ Tau & II & $<0.25$ & 180.5 & $\ldots$ & 2.5 & $\ldots$ \\
\hline GG Tau & II & 0.19 & 50.5 & $\cdots$ & 0.7 & $\cdots$ \\
\hline UZ Tau E & II & $<0.02$ & 65.0 & $\cdots$ & 0.6 & $\cdots$ \\
\hline UZ Tau W & III & $<0.02$ & 43.5 & $\ldots$ & 0.6 & $\ldots$ \\
\hline JH 112 & II & 0.21 & 16.0 & $\cdots$ & $\cdots$ & $\cdots$ \\
\hline L1551-55 & III & $<0.06$ & 1.8 & $\ldots$ & $\ldots$ & $\ldots$ \\
\hline GH Tau & II & 0.08 & 27.5 & $\ldots$ & $\ldots$ & $\ldots$ \\
\hline V807 Tau & II & 0.01 & 13.5 & $\ldots$ & $\ldots$ & $\ldots$ \\
\hline V830 Tau & III & $<0.02$ & 1.8 & $\ldots$ & $\ldots$ & $\cdots$ \\
\hline GI Tau & II & 0.19 & 14.5 & $\cdots$ & 0.8 & $\ldots$ \\
\hline GK Tau & II & 0.13 & 30.5 & $\cdots$ & 0.9 & $\cdots$ \\
\hline DL Tau & II & 0.33 & 101.0 & $\cdots$ & 2.2 & 0.4 \\
\hline HN Tau & II & 0.43 & 145.0 & 1.0 & 1.7 & 5.1 \\
\hline
\end{tabular}


Table 2. Continued

\begin{tabular}{|c|c|c|c|c|c|c|}
\hline Source Name & 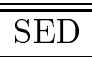 & $\overline{L_{F I R} / L_{\mathrm{b}}}$ & $\overline{\mathrm{H} \alpha}$ & [N II] 6584 & "He I 6678 & ["S II] $6717+6730$ \\
\hline IT Tau & II & $<0.05$ & 9.7 & $\cdots$ & 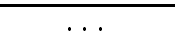 & $\cdots$ \\
\hline CI Tau & II & 0.17 & 76.5 & $\ldots$ & 1.0 & $\ldots$ \\
\hline DM Tau & II & 0.16 & 114.0 & $\ldots$ & 2.1 & $\ldots$ \\
\hline JH 108 & III & $<0.11$ & 3.3 & $\ldots$ & $\ldots$ & $\ldots$ \\
\hline HBC 407 & III & $<0.05$ & -1.8 & $\ldots$ & $\ldots$ & $\ldots$ \\
\hline AA Tau & II & 0.15 & 46.0 & $\cdots$ & 1.1 & $\ldots$ \\
\hline HO Tau & II & 0.33 & 101.5 & $\ldots$ & 2.2 & $\ldots$ \\
\hline FF Tau & III & 0.02 & 2.8 & $\ldots$ & $\ldots$ & $\cdots$ \\
\hline DN Tau & II & 0.09 & 18.0 & $\ldots$ & $\ldots$ & $\ldots$ \\
\hline HP Tau & II & 0.36 & 20.0 & $\cdots$ & $\cdots$ & $\cdots$ \\
\hline HP Tau/G2 & III & $<0.05$ & 2.8 & $\ldots$ & $\ldots$ & $\ldots$ \\
\hline HP Tau/G3 & III & $<0.01$ & 2.3 & $\ldots$ & $\ldots$ & $\ldots$ \\
\hline Haro 6-28 & I & 0.82 & 61.5 & $\cdots$ & $\cdots$ & $\cdots$ \\
\hline $\mathrm{LkCa} 14$ & III & 0.03 & 1.1 & $\ldots$ & $\ldots$ & $\ldots$ \\
\hline DO Tau & II & 0.24 & 101.0 & $\cdots$ & 2.0 & 1.4 \\
\hline HV Tau & III & $<0.02$ & 8.5 & $\ldots$ & $\ldots$ & $\ldots$ \\
\hline VY Tau & III & 0.14 & 7.3 & $\ldots$ & $\cdots$ & $\cdots$ \\
\hline $\mathrm{LkCa} 15$ & II & 0.11 & 18.5 & $\ldots$ & $\ldots$ & $\ldots$ \\
\hline JH 223 & II & $<0.17$ & 4.2 & $\ldots$ & $\ldots$ & $\ldots$ \\
\hline IW Tau & III & $<0.02$ & 4.0 & $\ldots$ & $\ldots$ & $\ldots$ \\
\hline CoKu Tau/4 & II & 0.33 & 2.8 & $\ldots$ & $\ldots$ & $\ldots$ \\
\hline LkHa332/G2 & III & $<0.02$ & 3.3 & $\ldots$ & $\cdots$ & $\cdots$ \\
\hline LkHa332/G1 & II & 0.08 & 4.9 & $\ldots$ & $\ldots$ & $\ldots$ \\
\hline V955 Tau & II & 0.15 & 20.0 & $\ldots$ & $\cdots$ & $\cdots$ \\
\hline DP Tau & II & 0.26 & 101.5 & 1.1 & 1.4 & 4.5 \\
\hline GO Tau & II & 0.17 & 78.0 & $\ldots$ & 1.3 & $\ldots$ \\
\hline DQ Tau & II & 0.21 & 102.0 & $\ldots$ & $\ldots$ & $\ldots$ \\
\hline Haro 6-37 & II & 0.11 & 13.0 & $\ldots$ & $\cdots$ & .. \\
\hline DR Tau & II & 0.28 & 78.0 & $\ldots$ & 1.5 & $\ldots$ \\
\hline DS Tau & II & 0.10 & 38.5 & $\ldots$ & 1.1 & $\ldots$ \\
\hline UY Aur & II & 0.36 & 63.5 & $\cdots$ & 0.9 & 1.8 \\
\hline St 34 & II & 0.15 & 90.4 & $\ldots$ & 2.4 & $\ldots$ \\
\hline GM Aur & II & 0.20 & 79.5 & $\ldots$ & $\ldots$ & $\ldots$ \\
\hline $\mathrm{LkCa} 19$ & III & $<0.01$ & 1.2 & $\ldots$ & $\ldots$ & $\ldots$ \\
\hline AB Aur & II & $<0.19$ & 44.0 & $\ldots$ & 0.5 & $\ldots$ \\
\hline SU Aur & II & 0.18 & 2.0 & $\cdots$ & $\ldots$ & $\ldots$ \\
\hline HBC 427 & III & 0.01 & 1.4 & $\ldots$ & $\ldots$ & $\ldots$ \\
\hline V836 Tau & III & 0.12 & 7.7 & $\ldots$ & $\ldots$ & $\ldots$ \\
\hline CIDA 8 & III & $<0.11$ & 79.0 & $\ldots$ & 2.7 & $\ldots$ \\
\hline CIDA 11 & III & $<0.13$ & 49.0 & $\cdots$ & $\cdots$ & $\cdots$ \\
\hline RW Aur & II & 0.18 & 75.5 & $\ldots$ & 1.2 & 1.4 \\
\hline
\end{tabular}




\section{FIGURE CAPTIONS}

Fig. 1.- Optical spectra of T Tauri stars. The degree of excitation increases clockwise from the weak emission line T Tauri star, LkCa 3, through the classical T Tauri stars, BP Tau and DP Tau, to the continuum + emission star DG Tau.

Fig. 2.- Spatially resolved spectra of Taurus pre-main-sequence stars. The left panels plot H $\alpha$ spectra for two class I sources, L1489 IRS (04016+2610) and HH31 IRS2 (04248+2612). The right panels plot [S II] spectra for L1527 IRS and HH30 IRS. All sources are very extended compared to the $1^{\prime \prime}-2^{\prime \prime}$ extent of an unresolved point source.

Fig. 3.- Optical spectra of four class I sources in Taurus-Auriga. All class I sources have strong emission from $\mathrm{H} \alpha$, [O I], and [S II]; some also have He I and Ca II triplet emission. Two systems - HH31 IRS2 and 04489+3042 - have the deep TiO absorption bands characteristic of M-type stars. A telluric absorption feature at $\lambda 7650$ is present in the spectra of L1489 IRS and $04264+2433$; this feature is blended with a TiO band in HH31 IRS2 and 04489+3042.

Fig. 4.- Optical spectra of two Taurus-Auriga class I sources. One class I source, 04158+2805, has deep $\mathrm{TiO}$ absorption bands and strong $\mathrm{H} \alpha,[\mathrm{O} \mathrm{I}]$, and [S II] emission lines. The spectrum of HH30 IRS is similar to other Herbig-Haro objects, with very intense emission from $\mathrm{H} \alpha,[\mathrm{O} \mathrm{I}],[\mathrm{N} \mathrm{II}]$, and [S II]. A telluric absorption feature at $\lambda 7650$ is present in the spectrum of HH30 IRS; this feature is blended with a TiO band in $04158+2805$.

Fig. 5.- Optical spectra of four class I sources in Taurus-Auriga. These objects have negligible continuum emission and very strong emission from $\mathrm{H} \alpha$, [O I], [N II], and [S II]. The emission line equivalent widths are comparable to those observed in Herbig-Haro objects.

Fig. 6.- TiO indices for field dwarfs and Taurus-Auriga pre-main-sequence stars. Several class I sources (diamonds) and heavily reddened $\mathrm{T}$ Tauri stars (plusses) have TiO band strengths comparable to those observed in T Tauri stars with negligible optical veiling (light filled triangles) and normal main-sequence stars (filled circles).

Fig. 7.- Frequency of [S II] emission among Taurus-Auriga pre-main-sequence stars. The [S II] emission frequency increases with the ratio of far-IR to bolometric luminosity, $L_{F I R} / L_{\mathrm{b}}$.

Fig. 8.- HR diagram for Taurus-Auriga pre-main-sequence stars. The filled circles are T Tauri stars from Kenyon \& Hartmann (1995). The crosses are class I sources from this study. The error bar in the upper right corner shows the typical uncertainty in luminosity and effective temperature for a class I source. Uncertainties for T Tauri stars are 33\%-50\% 
of the uncertainty for class I sources. The arrow indicates the change in $\log L$ and $\log \mathrm{T}_{\mathrm{e}}$ if a continuous source of emission veils the optical continuum. The dashed lines plot isochrones for the pre-main-sequence tracks of D'Antona \& Mazzitelli (1994) at times of $10^{5}, 10^{6}$, and $10^{7} \mathrm{yr}$ from top to bottom. The dot-dashed line is the stellar birthline from Stahler (1988). The solid lines are pre-main-sequence tracks for stars accreting from discs at $10^{-5} \mathrm{M}_{\odot} \mathrm{yr}^{-1}$ (thicker line) and $10^{-6} \mathrm{M}_{\odot} \mathrm{yr}^{-1}$ (thinner line). 

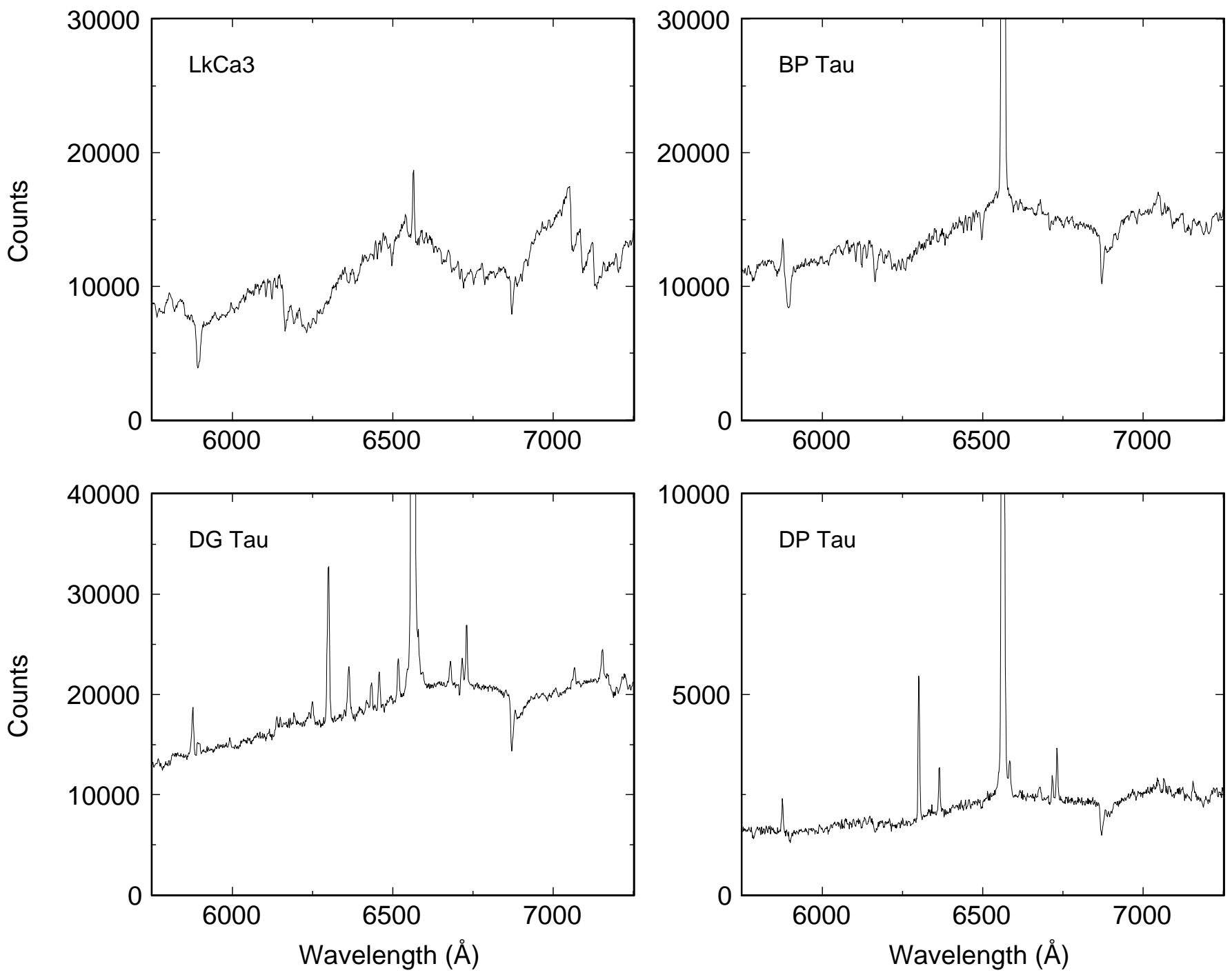

Figure 1 

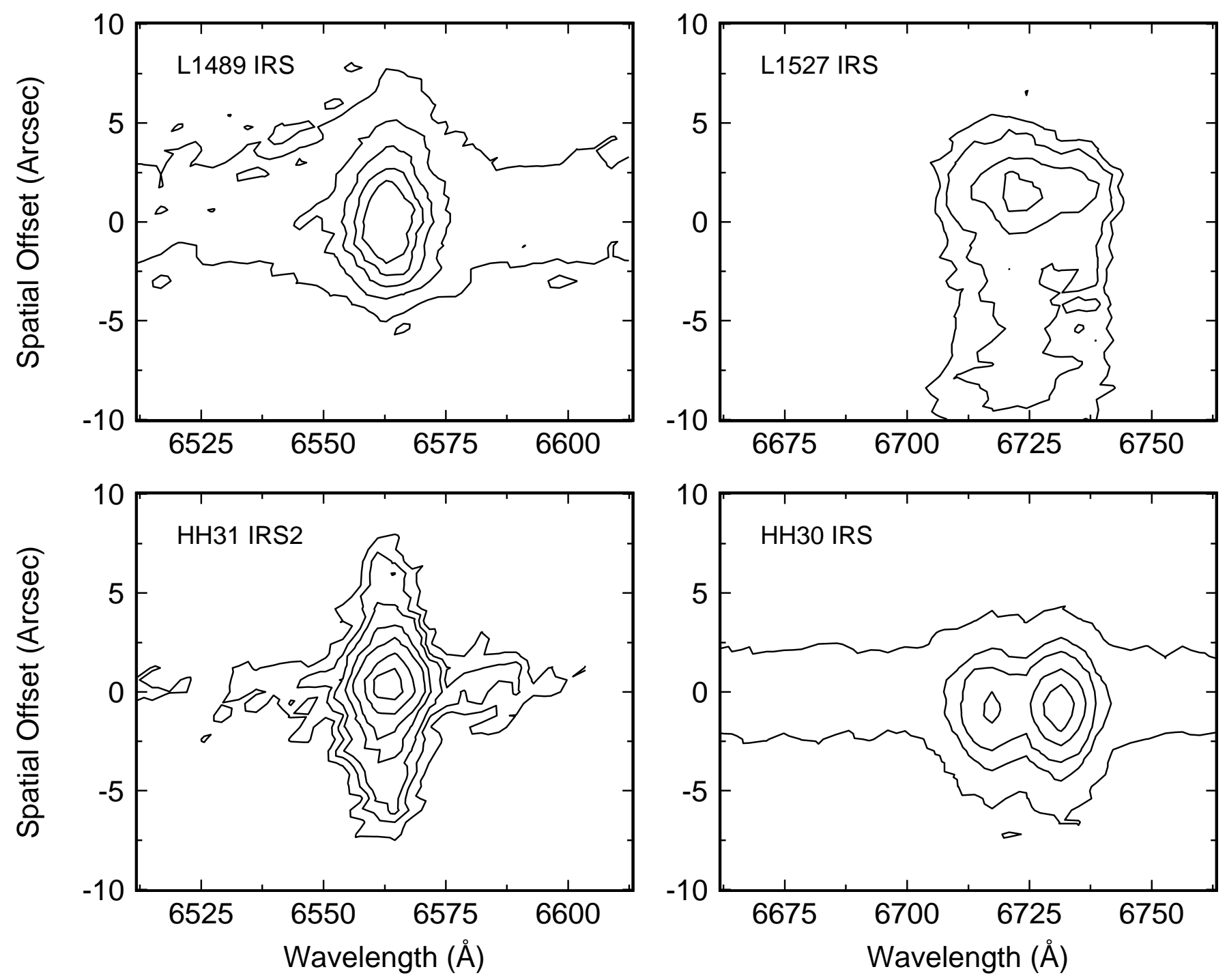

Figure 2 

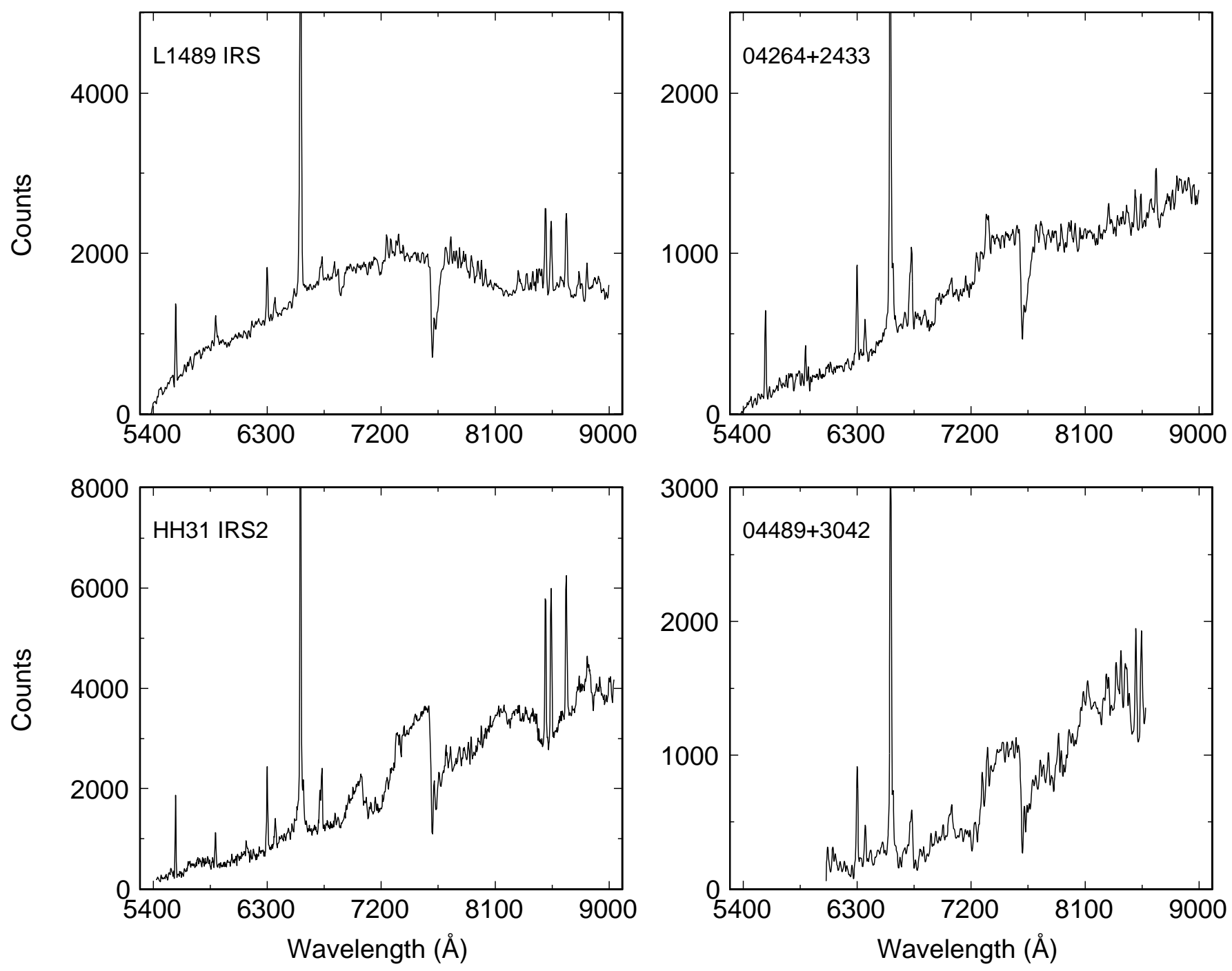

Figure 3 

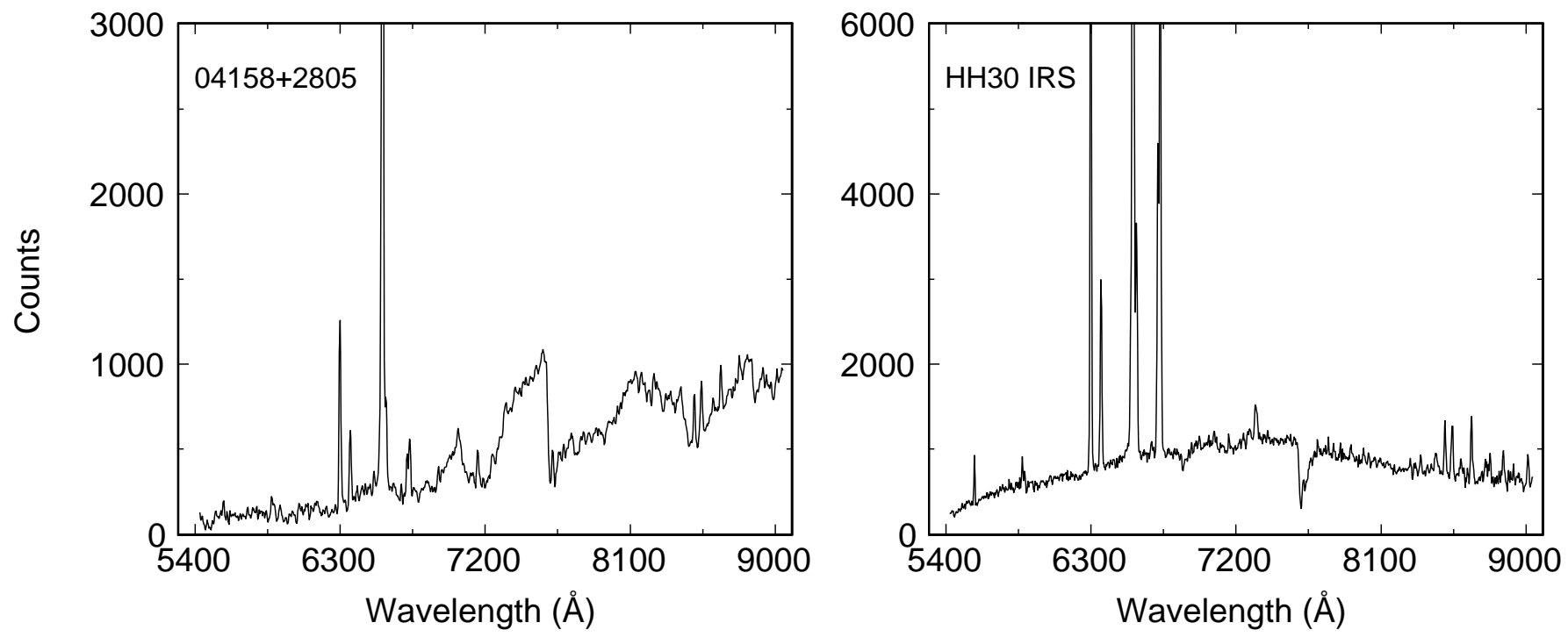

Figure 4 

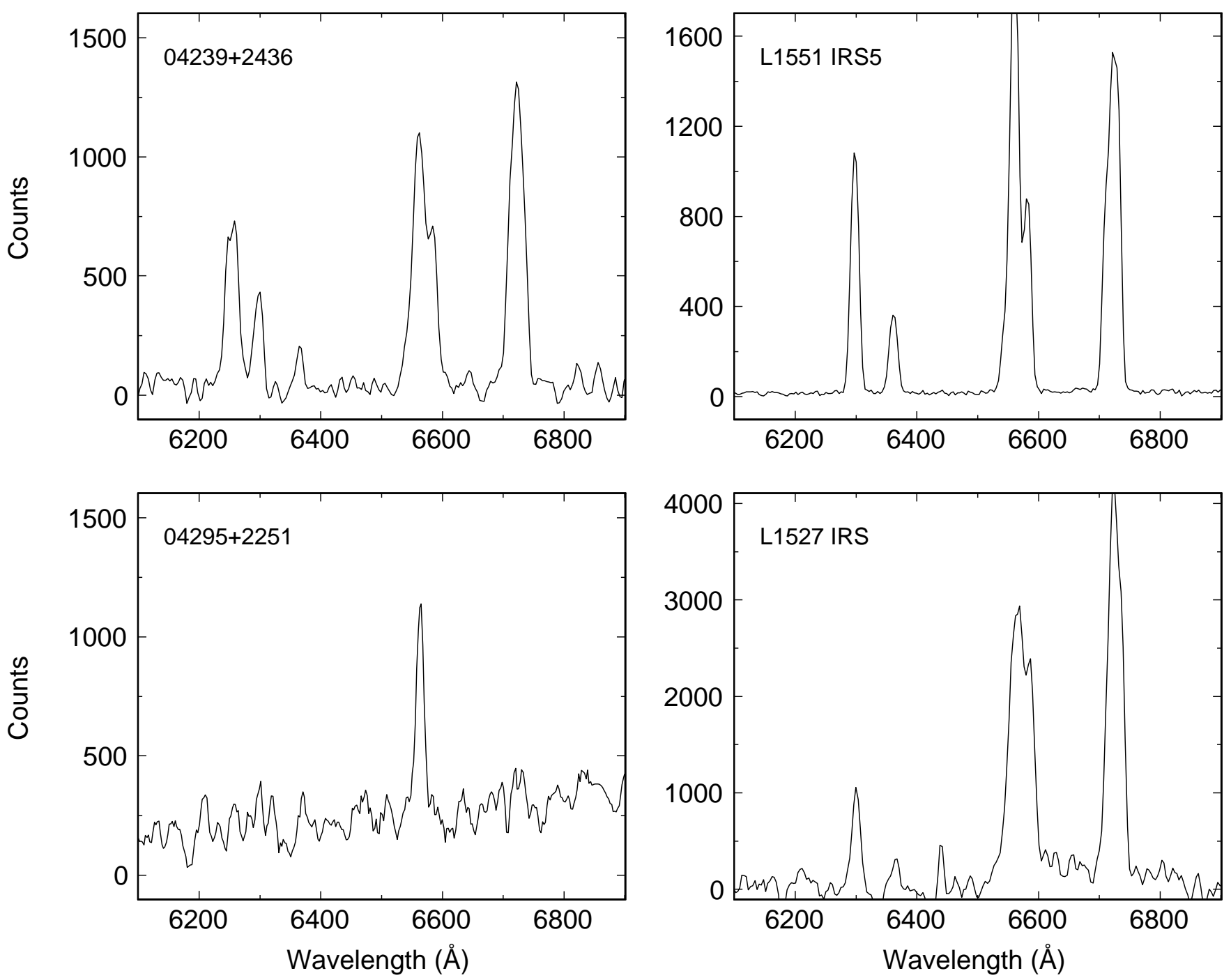

Figure 5 


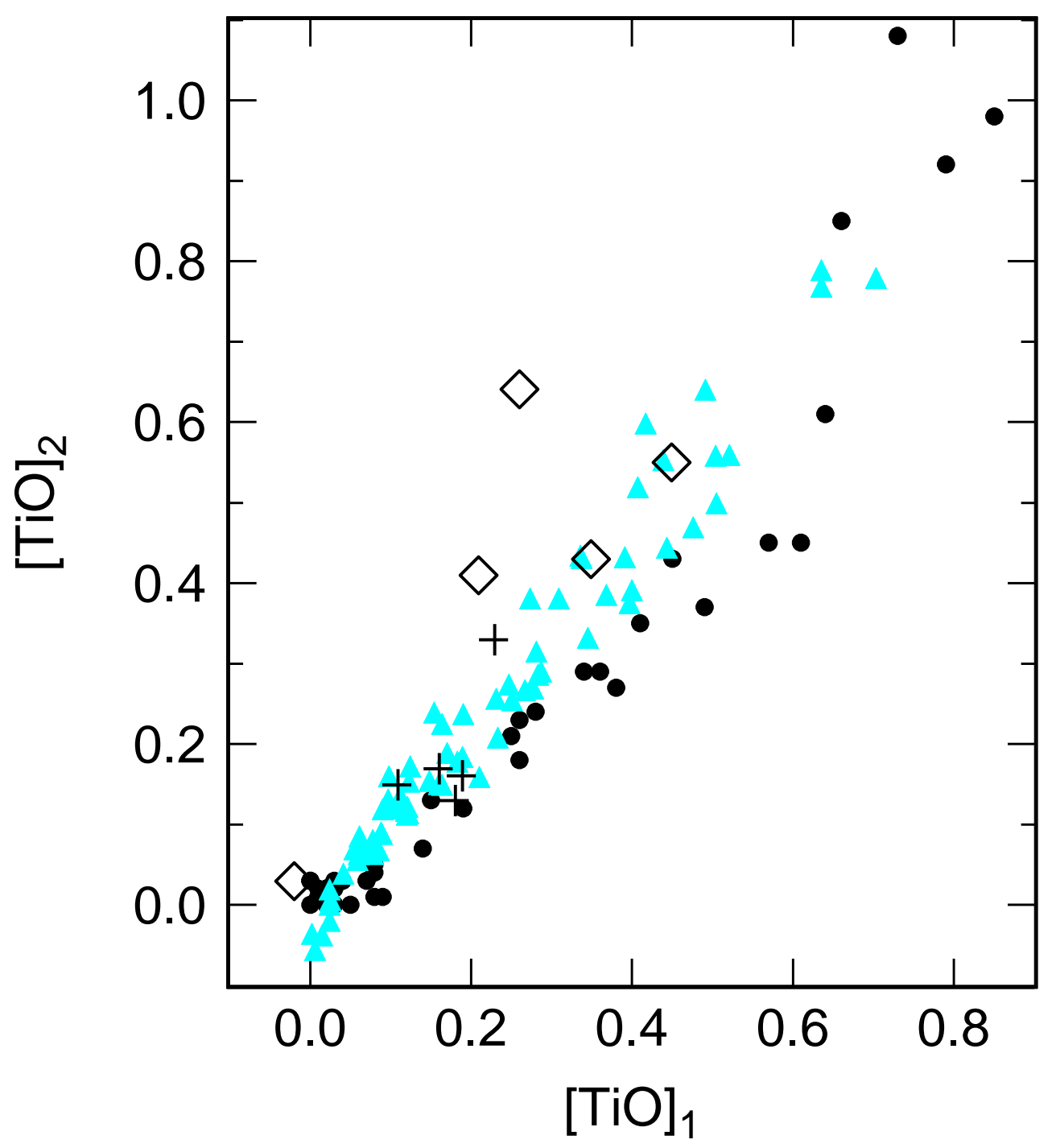

Figure 6 


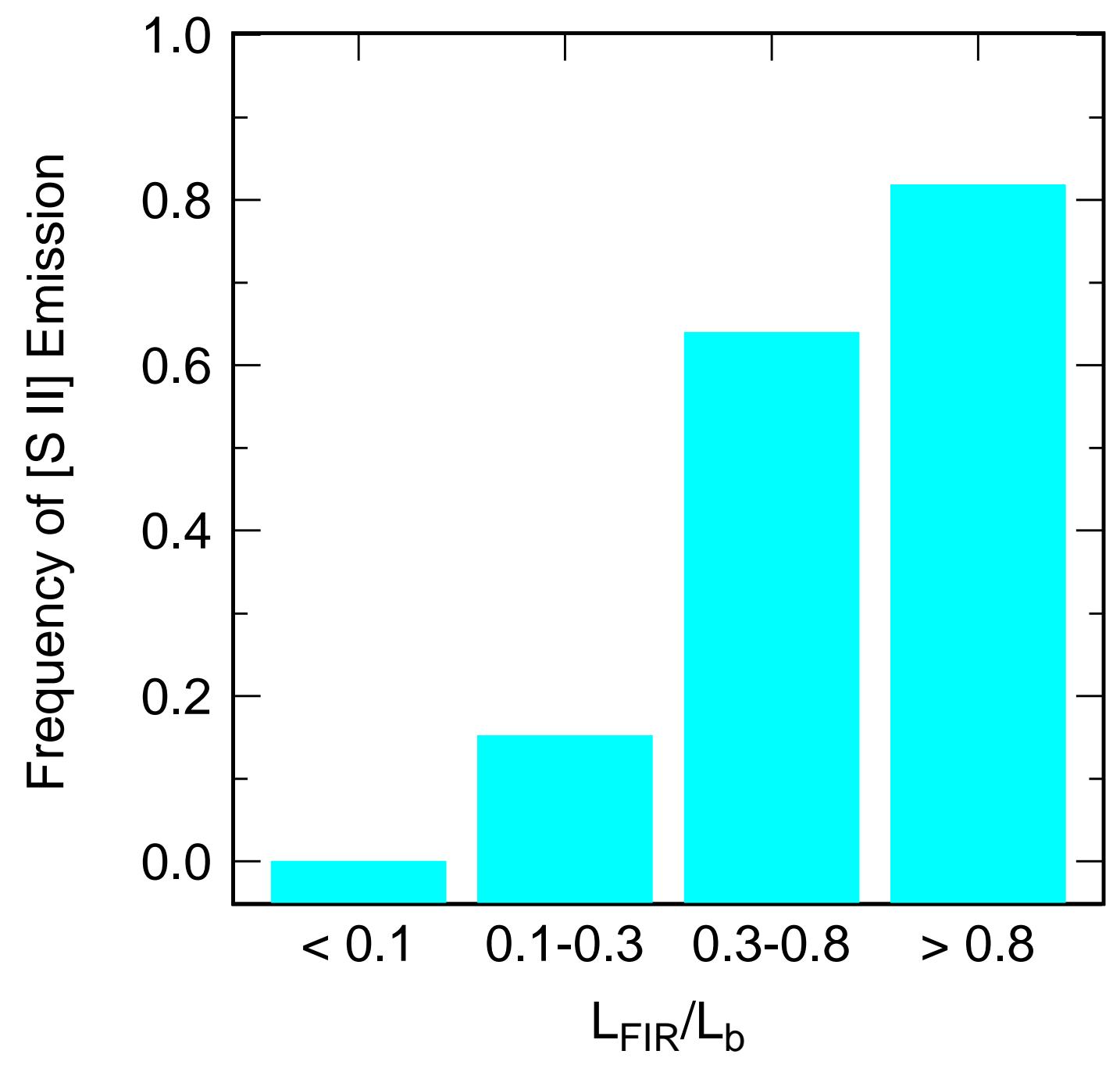

Figure 7 


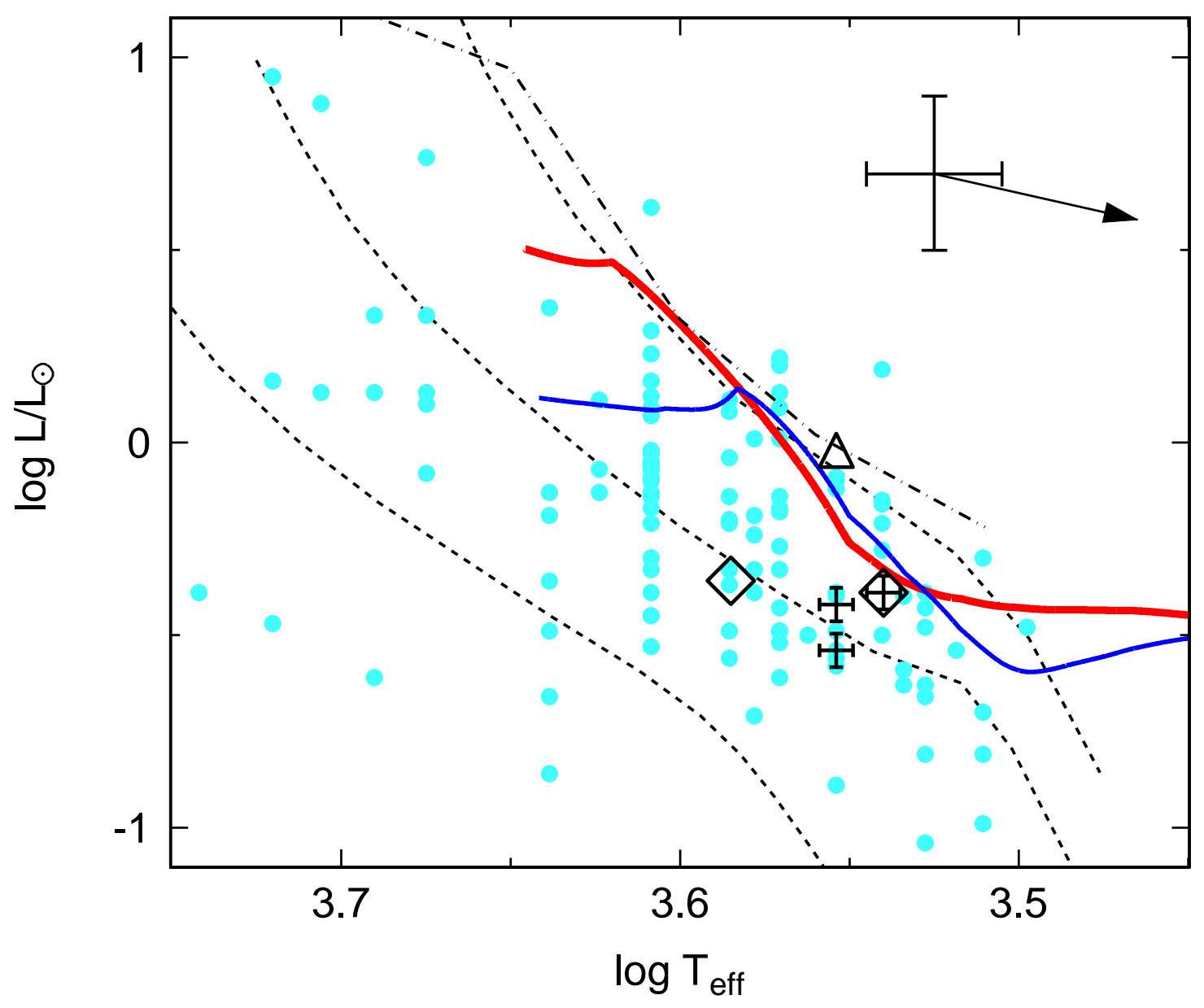

Figure 8 
Table 2. Emission line equivalent widths from FAST spectra

\begin{tabular}{|c|c|c|c|c|c|c|}
\hline Source Name & 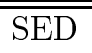 & $\bar{~} \overline{L_{F I R} / L_{\mathrm{b}}}$ & $\overline{\mathrm{H} \alpha}$ & [N II] 6584 & He I 6678 & [S II] $6717+6730$ \\
\hline HBC 351 & III & 0.05 & 2.8 & $\cdots$ & $\cdots$ & $\cdots$ \\
\hline HBC 352 & III & $<0.03$ & -2.2 & $\ldots$ & $\ldots$ & $\ldots$ \\
\hline HBC 353 & III & $<0.04$ & -2.1 & $\ldots$ & $\ldots$ & $\ldots$ \\
\hline HBC 354 & III & 0.14 & -1.2 & $\ldots$ & $\ldots$ & $\ldots$ \\
\hline HBC 355 & III & 0.07 & -1.2 & $\ldots$ & $\ldots$ & $\ldots$ \\
\hline HBC 356 & III & 0.06 & 0.8 & $\ldots$ & $\ldots$ & $\ldots$ \\
\hline HBC 357 & III & 0.10 & 1.1 & $\cdots$ & $\cdots$ & $\ldots$ \\
\hline HBC 358 & III & 0.04 & 10.0 & $\ldots$ & $\ldots$ & $\ldots$ \\
\hline HBC 359 & III & 0.04 & 5.4 & $\ldots$ & $\ldots$ & $\ldots$ \\
\hline HBC 360 & III & $<0.20$ & 6.7 & $\ldots$ & $\ldots$ & $\ldots$ \\
\hline HBC 361 & III & $<0.20$ & 6.4 & $\ldots$ & $\ldots$ & $\ldots$ \\
\hline HBC 362 & III & $<0.15$ & 6.6 & $\cdots$ & $\cdots$ & $\cdots$ \\
\hline $\mathrm{LkCa} 1$ & III & $<0.05$ & 3.4 & $\ldots$ & $\ldots$ & $\ldots$ \\
\hline Anon 1 & III & $<0.02$ & 2.5 & $\ldots$ & $\cdots$ & $\cdots$ \\
\hline $04108+2910$ & II & $<0.43$ & 37.5 & $\cdots$ & $\cdots$ & 2.0 \\
\hline V773 Tau & II & 0.10 & 2.8 & $\ldots$ & $\ldots$ & $\ldots$ \\
\hline FM Tau & II & 0.19 & 101.0 & $\cdots$ & 1.6 & $\cdots$ \\
\hline FN Tau & II & 0.31 & 16.0 & $\ldots$ & $\ldots$ & $\ldots$ \\
\hline CW Tau & II & 0.23 & 140.0 & $\cdots$ & 1.2 & 1.1 \\
\hline CIDA 1 & II & $<0.18$ & 149.0 & $\ldots$ & $\cdots$ & $\cdots$ \\
\hline $\mathrm{LkCa} 3$ & III & 0.01 & 2.7 & $\ldots$ & $\cdots$ & $\cdots$ \\
\hline FP Tau & III & 0.13 & 27.0 & 0.7 & $\ldots$ & $\ldots$ \\
\hline FO Tau & II & 0.15 & 116.0 & $\ldots$ & 1.5 & $\ldots$ \\
\hline CX Tau & II & 0.17 & 18.0 & $\ldots$ & $\ldots$ & $\ldots$ \\
\hline CIDA 2 & III & $<0.09$ & 6.8 & $\ldots$ & $\ldots$ & $\ldots$ \\
\hline $\mathrm{LkCa} 4$ & III & $<0.02$ & 3.2 & $\ldots$ & $\ldots$ & $\ldots$ \\
\hline CY Tau & II & 0.10 & 63.0 & $\cdots$ & 1.7 & $\cdots$ \\
\hline $\mathrm{LkCa} 5$ & III & 0.16 & 3.8 & $\cdots$ & $\cdots$ & $\cdots$ \\
\hline CIDA 3 & II & $<0.14$ & 10.5 & $\ldots$ & $\ldots$ & $\ldots$ \\
\hline V410 Tau & III & 0.01 & 2.3 & $\cdots$ & $\cdots$ & $\cdots$ \\
\hline DD Tau & II & 0.40 & 177.5 & $\ldots$ & 3.9 & 2.2 \\
\hline CZ Tau & II & 0.29 & 6.7 & $\ldots$ & $\ldots$ & $\ldots$ \\
\hline HBC 372 & III & $<0.15$ & -1.2 & $\ldots$ & $\ldots$ & $\ldots$ \\
\hline V892 Tau & II & 0.58 & 6.6 & $\ldots$ & $\cdots$ & $\cdots$ \\
\hline Hubble 4 & III & $\ldots$ & 3.9 & $\ldots$ & $\ldots$ & $\ldots$ \\
\hline HBC 376 & III & $<0.05$ & 1.9 & $\ldots$ & $\ldots$ & $\ldots$ \\
\hline FQ Tau & II & 0.15 & 81.0 & $\ldots$ & 3.0 & $\cdots$ \\
\hline BP Tau & II & 0.10 & 92.0 & $\ldots$ & 1.0 & $\ldots$ \\
\hline V819 Tau & III & 0.03 & 3.2 & $\ldots$ & $\ldots$ & $\ldots$ \\
\hline LkCa 7 & III & $<0.02$ & 3.9 & $\ldots$ & $\ldots$ & $\ldots$ \\
\hline $\mathrm{J} 2-157$ & III & $<0.50$ & 15.5 & $\ldots$ & $\cdots$ & $\cdots$ \\
\hline DE Tau & II & 0.11 & 59.0 & $\cdots$ & $\cdots$ & $\cdots$ \\
\hline
\end{tabular}


Table 2. Continued

\begin{tabular}{|c|c|c|c|c|c|c|}
\hline Source Name & SED & $\overline{L_{F I R} / L_{\mathrm{b}}}$ & $\overline{\mathrm{H} \alpha}$ & [N II] 6584 & ( He I 6678 & [S II] $6717+6730$ \\
\hline RY Tau & II & 0.25 & 13.0 & $\cdots$ & $\cdots$ & $\cdots$ \\
\hline HD 283572 & III & $<0.02$ & -1.6 & $\ldots$ & $\ldots$ & $\ldots$ \\
\hline FS Tau & II & 0.50 & 76.0 & 5.5 & 1.8 & 8.9 \\
\hline T Tau & II & 0.20 & 40.5 & $\cdots$ & 1.7 & 1.7 \\
\hline IP Tau & II & 0.16 & 10.5 & $\ldots$ & $\ldots$ & $\ldots$ \\
\hline $\mathrm{J} 1-4872$ & III & $<0.04$ & 2.9 & $\cdots$ & $\cdots$ & $\cdots$ \\
\hline FV Tau & II & 0.16 & 20.0 & $\cdots$ & $\cdots$ & $\cdots$ \\
\hline DF Tau & II & 0.08 & 54.5 & $\ldots$ & 1.7 & $\ldots$ \\
\hline DG Tau & II & 0.60 & 125.0 & 0.7 & 1.0 & 2.4 \\
\hline HBC 388 & III & 0.02 & -1.6 & $\ldots$ & $\ldots$ & $\ldots$ \\
\hline DH Tau & II & 0.12 & 38.5 & $\ldots$ & 1.4 & 0.6 \\
\hline DI Tau & II & 0.06 & 2.0 & $\ldots$ & $\ldots$ & $\ldots$ \\
\hline IQ Tau & II & 0.12 & 7.7 & $\cdots$ & $\cdots$ & $\cdots$ \\
\hline FX Tau & II & 0.16 & 14.5 & $\cdots$ & $\cdots$ & $\cdots$ \\
\hline DK Tau & II & 0.18 & 49.5 & $\cdots$ & 1.0 & 1.0 \\
\hline ZZ Tau & III & 0.07 & 14.0 & $\cdots$ & $\cdots$ & $\cdots$ \\
\hline JH 56 & III & $<0.06$ & 2.2 & $\ldots$ & $\ldots$ & $\cdots$ \\
\hline V927 Tau & III & $<0.05$ & 10.0 & $\cdots$ & $\cdots$ & $\cdots$ \\
\hline HBC 392 & III & 0.06 & 1.1 & $\cdots$ & $\cdots$ & $\cdots$ \\
\hline HL Tau & II & 0.77 & 48.0 & $\cdots$ & $\cdots$ & 3.6 \\
\hline XZ Tau & II & 0.61 & 175.0 & $\ldots$ & 1.4 & 2.2 \\
\hline HK Tau & $\mathrm{I} / \mathrm{II}$ & 0.27 & 53.5 & $\ldots$ & 0.4 & $\cdots$ \\
\hline V710 Tau A & II & 0.08 & 33.5 & $\cdots$ & $\cdots$ & $\cdots$ \\
\hline V710 Tau B & II & 0.14 & 4.3 & $\cdots$ & $\cdots$ & $\cdots$ \\
\hline L1551-51 & III & $<0.04$ & 1.5 & $\cdots$ & $\cdots$ & $\cdots$ \\
\hline V928 Tau & III & $<0.04$ & 1.8 & $\cdots$ & $\cdots$ & $\cdots$ \\
\hline V827 Tau & III & $<0.04$ & 4.4 & $\cdots$ & $\cdots$ & $\cdots$ \\
\hline V826 Tau & III & 0.02 & 3.5 & $\cdots$ & $\cdots$ & $\cdots$ \\
\hline FY Tau & II & 0.12 & 73.0 & $\ldots$ & 0.2 & $\cdots$ \\
\hline FZ Tau & II & $<0.25$ & 180.5 & $\cdots$ & 2.5 & $\cdots$ \\
\hline GG Tau & II & 0.19 & 50.5 & $\cdots$ & 0.7 & $\cdots$ \\
\hline UZ Tau E & II & $<0.02$ & 65.0 & $\cdots$ & 0.6 & $\cdots$ \\
\hline UZ Tau W & III & $<0.02$ & 43.5 & $\cdots$ & 0.6 & $\cdots$ \\
\hline JH 112 & II & 0.21 & 16.0 & $\cdots$ & $\cdots$ & $\cdots$ \\
\hline L1551-55 & III & $<0.06$ & 1.8 & $\cdots$ & $\cdots$ & $\cdots$ \\
\hline GH Tau & II & 0.08 & 27.5 & $\ldots$ & $\cdots$ & $\cdots$ \\
\hline V807 Tau & II & 0.01 & 13.5 & $\cdots$ & $\cdots$ & $\cdots$ \\
\hline V830 Tau & III & $<0.02$ & 1.8 & $\cdots$ & $\cdots$ & $\cdots$ \\
\hline GI Tau & II & 0.19 & 14.5 & $\ldots$ & 0.8 & $\cdots$ \\
\hline GK Tau & II & 0.13 & 30.5 & $\cdots$ & 0.9 & $\cdots$ \\
\hline DL Tau & II & 0.33 & 101.0 & $\cdots$ & 2.2 & 0.4 \\
\hline HN Tau & II & 0.43 & 145.0 & 1.0 & 1.7 & 5.1 \\
\hline
\end{tabular}


Table 2. Continued

\begin{tabular}{|c|c|c|c|c|c|c|}
\hline Source Name & 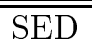 & $\overline{\overline{L_{F I R}} / L_{\mathrm{b}}}$ & $\overline{\mathrm{H} \alpha}$ & [N II] 6584 & "He I 6678 & $\overline{\text { [S II] } 6717+6730}$ \\
\hline IT Tau & II & $<0.05$ & 9.7 & $\cdots$ & $\cdots$ & $\cdots$ \\
\hline CI Tau & II & 0.17 & 76.5 & $\ldots$ & 1.0 & $\ldots$ \\
\hline DM Tau & II & 0.16 & 114.0 & $\cdots$ & 2.1 & $\cdots$ \\
\hline JH 108 & III & $<0.11$ & 3.3 & $\cdots$ & $\cdots$ & $\cdots$ \\
\hline HBC 407 & III & $<0.05$ & -1.8 & $\ldots$ & $\ldots$ & $\ldots$ \\
\hline AA Tau & II & 0.15 & 46.0 & $\cdots$ & 1.1 & $\cdots$ \\
\hline HO Tau & II & 0.33 & 101.5 & $\ldots$ & 2.2 & $\ldots$ \\
\hline FF Tau & III & 0.02 & 2.8 & $\ldots$ & $\cdots$ & $\ldots$ \\
\hline DN Tau & II & 0.09 & 18.0 & $\ldots$ & $\ldots$ & $\ldots$ \\
\hline HP Tau & II & 0.36 & 20.0 & $\ldots$ & $\cdots$ & $\cdots$ \\
\hline HP Tau/G2 & III & $<0.05$ & 2.8 & ... & $\ldots$ & $\ldots$ \\
\hline HP Tau/G3 & III & $<0.01$ & 2.3 & $\ldots$ & $\ldots$ & $\ldots$ \\
\hline Haro 6-28 & I & 0.82 & 61.5 & $\ldots$ & $\ldots$ & $\ldots$ \\
\hline $\mathrm{LkCa} 14$ & III & 0.03 & 1.1 & $\ldots$ & $\ldots$ & $\ldots$ \\
\hline DO Tau & II & 0.24 & 101.0 & $\cdots$ & 2.0 & 1.4 \\
\hline HV Tau & III & $<0.02$ & 8.5 & $\ldots$ & $\cdots$ & $\cdots$ \\
\hline VY Tau & III & 0.14 & 7.3 & $\ldots$ & $\cdots$ & $\cdots$ \\
\hline $\mathrm{LkCa} 15$ & II & 0.11 & 18.5 & $\cdots$ & $\cdots$ & $\cdots$ \\
\hline JH 223 & II & $<0.17$ & 4.2 & $\cdots$ & $\cdots$ & $\cdots$ \\
\hline IW Tau & III & $<0.02$ & 4.0 & $\cdots$ & $\cdots$ & $\cdots$ \\
\hline $\mathrm{CoKu} \mathrm{Tau} / 4$ & II & 0.33 & 2.8 & $\cdots$ & $\cdots$ & $\cdots$ \\
\hline LkHa332/G2 & III & $<0.02$ & 3.3 & $\cdots$ & $\cdots$ & $\cdots$ \\
\hline LkHa332/G1 & II & 0.08 & 4.9 & $\ldots$ & $\ldots$ & $\ldots$ \\
\hline V955 Tau & II & 0.15 & 20.0 & $\ldots$ & $\cdots$ & $\cdots$ \\
\hline DP Tau & II & 0.26 & 101.5 & 1.1 & 1.4 & 4.5 \\
\hline GO Tau & II & 0.17 & 78.0 & $\ldots$ & 1.3 & $\ldots$ \\
\hline DQ Tau & II & 0.21 & 102.0 & $\cdots$ & $\cdots$ & $\cdots$ \\
\hline Haro 6-37 & II & 0.11 & 13.0 & $\ldots$ & $\cdots$ & $\ldots$ \\
\hline DR Tau & II & 0.28 & 78.0 & $\cdots$ & 1.5 & $\cdots$ \\
\hline DS Tau & II & 0.10 & 38.5 & $\cdots$ & 1.1 & $\cdots$ \\
\hline UY Aur & II & 0.36 & 63.5 & $\cdots$ & 0.9 & 1.8 \\
\hline St 34 & II & 0.15 & 90.4 & $\ldots$ & 2.4 & $\cdots$ \\
\hline GM Aur & II & 0.20 & 79.5 & $\cdots$ & $\cdots$ & $\cdots$ \\
\hline $\mathrm{LkCa} 19$ & III & $<0.01$ & 1.2 & $\ldots$ & $\cdots$ & $\ldots$ \\
\hline AB Aur & II & $<0.19$ & 44.0 & $\cdots$ & 0.5 & $\cdots$ \\
\hline SU Aur & II & 0.18 & 2.0 & $\cdots$ & $\cdots$ & $\cdots$ \\
\hline HBC 427 & III & 0.01 & 1.4 & $\ldots$ & $\cdots$ & $\ldots$ \\
\hline V836 Tau & III & 0.12 & 7.7 & $\cdots$ & $\cdots$ & $\cdots$ \\
\hline CIDA 8 & III & $<0.11$ & 79.0 & $\ldots$ & 2.7 & $\cdots$ \\
\hline CIDA 11 & III & $<0.13$ & 49.0 & $\cdots$ & $\cdots$ & $\cdots$ \\
\hline RW Aur & II & 0.18 & 75.5 & $\ldots$ & 1.2 & 1.4 \\
\hline
\end{tabular}

